\title{
МЕТОД Н. П. КУПЦОВА ПОСТРОЕНИЯ ЭКСТРЕМАЛЬНОЙ ФУНКЦИИ В НЕРАВЕНСТВЕ МЕЖДУ РАВНОМЕРНЫМИ НОРМАМИ ПРОИЗВОДНЫХ ФУНКЦИЙ НА ПОЛУОСИ
}

\section{В. Г. Тимофеев}

На классе $L_{\infty}^{4}\left(\mathbb{R}_{+}\right)$функций $f \in C\left(\mathbb{R}_{+}\right)$, имеющих локально-абсолютно непрерывную производную третьего порядка на полупрямой $\mathbb{R}_{+}$и таких, что $f^{(4)} \in L_{\infty}\left(\mathbb{R}_{+}\right)$, исследуется экстремальная функция в точных неравенствах

$$
\left\|f^{(j)}\right\| \leq C_{4, j}\left(\mathbb{R}_{+}\right)\|f\|^{1-j / 4}\left\|f^{(4)}\right\|^{j / 4}, \quad j=\overline{1,3}, \quad f \in L_{\infty}^{4}\left(\mathbb{R}_{+}\right) .
$$

Изложен неопубликованный ранее метод Н. П. Купцова построения экстремальной функции, являющейся идеальным сплайном четвертой степени. Метод итерационный, он позволяет находить узлы и коэффициенты сплайна, содержит алгоритм вычисления величин $C_{4, j}\left(\mathbb{R}_{+}\right)$. Предложенный подход отличается от подхода Шёнберга и Каваретты (1970), позволяет более глубоко понять структуру задачи.

Ключевые слова: неравенство между нормами производных функций, четыре раза дифференцируемые функции, равномерная норма, полуось.

V. G. Timofeev. N.P. Kuptsov's method for the construction of an extremal function in an inequality between uniform norms of derivatives of functions on the half-line.

On the class $L_{\infty}^{4}\left(\mathbb{R}_{+}\right)$of functions $f \in C\left(\mathbb{R}_{+}\right)$having a locally absolutely continuous third-order derivative on the half-line $\mathbb{R}_{+}$and such that $f^{(4)} \in L_{\infty}\left(\mathbb{R}_{+}\right)$, we study an extremal function in the exact inequalities

$$
\left\|f^{(j)}\right\| \leq C_{4, j}\left(\mathbb{R}_{+}\right)\|f\|^{1-j / 4}\left\|f^{(4)}\right\|^{j / 4}, \quad j=\overline{1,3}, \quad f \in L_{\infty}^{4}\left(\mathbb{R}_{+}\right) .
$$

We present N.P. Kuptsov's earlier unpublished method for the construction of an extremal function, which is an ideal spline of the fourth degree. The method is iterative; it finds the knots and coefficients of the spline and calculates the values $C_{4, j}\left(\mathbb{R}_{+}\right)$. The proposed approach differs from the approach of Schoenberg and Cavaretta (1970) and allows to understand the structure of the problem more deeply.

Keywords: inequality between norms of derivatives of functions, four times differentiable functions, uniform norm, half-line.

MSC: 26D10

DOI: $10.21538 / 0134-4889-2019-25-2-220-239$

\section{1. Введение}

Обозначим через $L_{\infty}\left(\mathbb{R}_{+}\right)$пространство измеримых существенно ограниченных на полуоси $\mathbb{R}_{+}=[0, \infty)$ функций $f$ с нормой

$$
\|f\|=\operatorname{ess} \sup _{t \in \mathbb{R}_{+}}|f(t)|
$$

а через $L_{\infty}^{n}\left(\mathbb{R}_{+}\right), n \in \mathbb{N},-$ пространство функций $f \in L_{\infty}\left(\mathbb{R}_{+}\right)$, имеющих на $\mathbb{R}_{+}$локально абсолютно непрерывную производную $f^{(n-1)}$ и таких, что $f^{(n)} \in L_{\infty}\left(\mathbb{R}_{+}\right)$.

Для заданных положительных чисел $A$ и $B, k=1,2, \ldots,(n-1)$ и функций $f \in L_{\infty}^{n}\left(\mathbb{R}_{+}\right)$ хорошо известна следующая восходящая к Э. Ландау [1], экстремальная задача

$$
\left\|f^{(k)}\right\| \rightarrow \sup , \quad\|f\| \leq A, \quad\left\|f^{(n)}\right\| \leq B
$$


В нашем случае эта задача эквивалентна отысканию точной константы в неравенстве

$$
\left\|f^{(k)}\right\| \leq C_{n, k}\left(\mathbb{R}_{+}\right)\|f\|^{1-k / n} \cdot\left\|f^{(n)}\right\|^{k / n}, \quad f \in L_{\infty}^{n}\left(\mathbb{R}_{+}\right) .
$$

Отметим, что в случае оси $\mathbb{R}=(-\infty ; \infty)$ такая задача для всех $1 \leq k<n$ была полностью решена А.Н. Колмогоровым [2]. На полуоси задача оказалась существенно сложнее.

Э. Ландау [1] доказал, что $C_{2,1}\left(\mathbb{R}_{+}\right)=2$. А.П. Маторин [3] установил, что при всех $n, k$, $k<n$,

$$
C_{n, k}\left(\mathbb{R}_{+}\right) \leq \frac{T_{n}^{(k)}(1)}{\left\|T_{n}\right\|^{1-k / n}},
$$

где

$$
T_{n}(x)=\frac{1}{2^{n-1} n !} \cos (n \arccos x), \quad x \in[-1,1],
$$

есть многочлен Чебышева первого рода. В случаях $n=2, k=1$ и $n=3, k=1,2$ неравенство (1.1) обращается в равенство.

Наилучшие на данный момент двусторонние оценки для констант $C_{n, k}\left(\mathbb{R}_{+}\right)$были получены С. Б. Стечкиным [4]. Для $n \geq 4$ задача о точных константах $C_{n, k}\left(\mathbb{R}_{+}\right)$была решена И. Шёнбергом и А. Кавареттой [5;6]. Проведенная в [5;6] схема рассуждений оставляла открытыми вопросы структуры экстремального сплайна и связи точных неравенств с родственными задачами $[7 ; 8]$ теории приближений.

Структура экстремальной функции для $n=4$ изучается в настоящей работе методом Николая Петровича Купцова; он отличен от метода работ [5;6]. Основные идеи, приведенного ниже доказательства, были изложены им в 1974 г. на Объединенном семинаре механикоматематического факультета Саратовского государственного университета. В 1994 г. Николай Петрович ушел из жизни. До настоящего момента его результат не был опубликован. Автор данной статьи, являясь учеником Н.П.Купцова, посчитал возможным и полезным, опубликовать результат Николая Петровича, дополнив некоторые отсутствующие его этапы доказательства. Исследования, относящиеся к случаям $n=5,6$, проводились автором ранее в [9;10] с применением метода Н. П. Купцова.

Пусть $L_{\infty}^{4}\left(\mathbb{R}_{+}\right)$- множество функций $f$, непрерывных на $\mathbb{R}_{+}=[0, \infty)$ вместе с производными до 3-го порядка включительно и таких, что

1) $\sup _{0 \leq t<\infty}|f(t)|<\infty$

2) $f^{\prime \prime \prime}(t)$ удовлетворяет на $[0, \infty)$ условию Липшица порядка 1.

Для всякой функции $f \in L_{\infty}^{4}\left(\mathbb{R}_{+}\right)$существует почти всюду на $[0, \infty)$ производная 4-го порядка, и при этом $\operatorname{ess} \sup \left\{\left|f^{I V}(t)\right|: 0 \leq t<\infty\right\}<\infty$.

Пусть $\Omega_{4}[0, \infty)$ есть множество функций из $L_{\infty}^{4}\left(\mathbb{R}_{+}\right)$, для которых $\|f\| \leq 1$ и $\left\|f^{I V}\right\| \leq 2 \cdot 4$ !. Положим

$$
\mu_{4 j}=\sup _{f \in \Omega_{4}[0, \infty)}\left\|f^{(j)}\right\|, \quad j=\overline{1,3} .
$$

Известно, что $\mu_{4 j}<\infty, j=\overline{1,3}$.

Обычным способом можно показать (см., например, [4]), что для всякой функции $f$ из $L_{\infty}^{4}\left(\mathbb{R}_{+}\right)$справедливы следующие точные неравенства:

$$
\left\|f^{(j)}\right\| \leq \frac{\mu_{4 j}}{(\sqrt[4]{48})^{j}}\|f\|^{(4-j) / 4}\left\|f^{I V}\right\|^{j / 4}, \quad j=\overline{1,3}
$$

Основная цель данной статьи состоит в том, чтобы выяснить структуру экстремальной функции неравенства (1.2) и указать способ вычисления величин $\mu_{4 j}, j=\overline{1,3}$. Для этого будет построена функция $\varphi \in \Omega_{4}[0, \infty)$, обладающая свойствами:

$$
\left\|\varphi^{(j)}\right\|=\mu_{4 j}, \quad j=\overline{1,3} .
$$




\section{2. Об одном специальном отображении}

Обозначим через $G^{\prime}$ открытый прямоугольник в плоскости переменных $\theta, L$, определяемый неравенствами: $0<\theta<1,1<L<\frac{2}{\sqrt[4]{10}}$ (рис. 1 ).

Рассмотрим отображение замкнутого прямоугольника $\bar{G}^{\prime}$, задаваемое формулами

$$
\left\{\begin{array}{l}
x=\frac{L^{4}\left(1-4 \theta^{3}+2 \theta^{4}\right)-1}{L^{3}}, \\
y=\frac{3-L^{4}\left(1-8 \theta^{3}+6 \theta^{4}\right)}{L^{2}} .
\end{array}\right.
$$

Открытая область $G$, являющаяся образом $G^{\prime}$ при отображении (2.1), схематически изображена на рис. 2.

Докажем, что отображение $\bar{G}^{\prime}$ на $\bar{G}$ взаимнооднозначно. В области $0<\theta<1$ и $1 \leq L \leq \frac{2}{\sqrt[4]{10}}$

$$
\frac{\partial x}{\partial L}=1-4 \theta^{3}+2 \theta^{4}+\frac{3}{L^{4}}>0, \quad \frac{\partial x}{\partial \theta}=4 L \theta^{2}(2 \theta-3)<0 .
$$

Отсюда следует, что каждая из сторон прямоугольника $\bar{G}^{\prime}$ отображается взаимнооднозначно на соответствующий участок границы $\bar{G}$. При $0<\theta<1$ и $1 \leq L \leq \frac{2}{\sqrt[4]{10}}$ якобиан отображения (2.1) имеет вид

$$
\frac{\partial(x, y)}{\partial(\theta, L)}=8 \theta^{3} L^{2}\left[\frac{3}{L^{4}}+1-12 \theta^{2}+16 \theta^{3}-6 \theta^{4}\right]>0 .
$$

Используя теорему Л. Д. Кудрявцева [11, теорема 13] (см. доказательство в [12, теорема 1.13]), получаем, что отображение (2.1) взаимнооднозначно. Это означает, что для всех точек области $\bar{G}$ можно ввести криволинейные координаты $L$ и $\theta$ (рис. 3 ).

Рассмотрим некоторые важные для дальнейшего построения точки и линии плоскости $x, y$. Обозначим через $M \tilde{Q}$ кривую, симметричную кривой $M Q$ относительно оси оy. Пусть $U-$ точка пересечения кривых $M \tilde{Q}$ и $P Q$. Обозначим кривую, соответствующую $\theta=\frac{1}{2}$, через $V T$. $F$ - точка пересечения кривых $V T$ и $M U$.

Рассмотрим два элементарных преобразования области $\bar{G}$. Пусть преобразование $K$ ставит в соответствие точке $a$ с криволинейными координатами $(\theta, L)$ точку $K a$ с координатами $(1-$ $\theta, L)$. Пусть $S$ - преобразование симметрии относительно оси оу.

Преобразование $K$ всякую точку области $\bar{G}$ переводит в точку той же области, а преобразование $S$ может вывести точку за пределы этой области.

Лемма 1. Точка $T=\left\{0 ; \frac{6}{\sqrt{10}}\right\}$ является единственной неподвиюной точкой преобразования $S K$, принадлежсащей области $\bar{G}$.

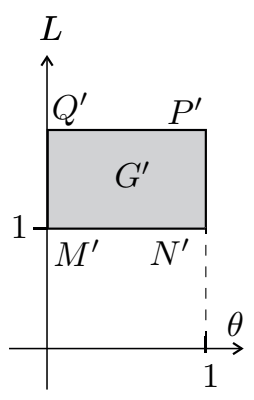

Рис. 1

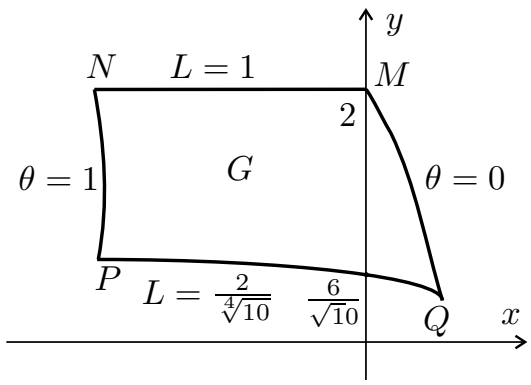

Рис. 2

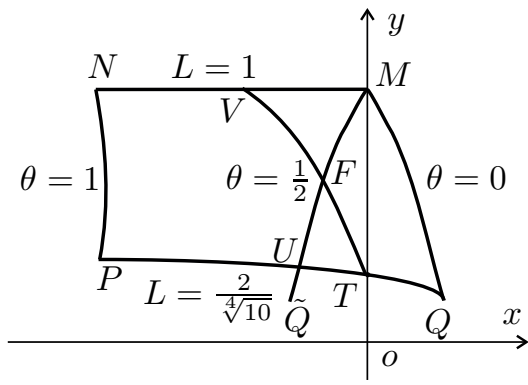

Рис. 3 
Д о к а з а т е л ь с т в о. Пусть $\zeta_{0}-$ неподвижная точка и $\theta$ и $L-$ ее криволинейные координаты. Тогда равенство $\zeta_{0}=S K \zeta_{0}$ дает систему

$$
\left\{\begin{array}{l}
\frac{L^{4}\left[1-4 \theta^{3}+2 \theta^{4}\right]-1}{L^{3}}=\frac{1-L^{4}\left[1-4(1-\theta)^{3}+2(1-\theta)^{4}\right]}{L^{3}} \\
\frac{3-L^{4}\left[1-8 \theta^{3}+6 \theta^{4}\right]}{L^{2}}=\frac{3-L^{4}\left[1-8(1-\theta)^{3}+6(1-\theta)^{4}\right]}{L^{2}} .
\end{array}\right.
$$

Из второго уравнения системы $(2.2)$ вытекает, что $1-8 \theta^{3}+6 \theta^{4}=1-8(1-\theta)^{3}+6(1-\theta)^{4}$. Единственным решением последнего уравнения на отрезке $[0,1]$ является точка $\theta=\frac{1}{2}$. Отсюда из первого уравнения системы $(2.2)$ получаем $\frac{2}{L^{3}}-\frac{5}{4} L=0$. Таким образом, криволинейные координаты неподвижной точки $\theta=\frac{1}{2}, L=\frac{2}{\sqrt[4]{10}}$, следовательно, $T=\left\{0 ; \frac{6}{\sqrt{10}}\right\}$.

Лемма 2. Для всякой точки z замкнутого четырехугольника $V M Q T$, отличной от точ$\kappa и T$, всегда найдется такое натуральное $n$, что $(S K)^{n} z \notin \bar{G}$. Если точка $z \in V N P T$ такал, что $(S K)^{n} z \in \bar{G}$ для любых натуральных $n$, то все точки последовательности $(S K)^{n} z$ принадлежат VNPT, и последовательность сходится к точке $T$.

Д о к а з а т е л ь с т в о. Для точки $z \in \bar{G}$ обозначим через $z_{k}=\left\{A_{k}, B_{k}\right\}$ точки множества $\bar{G}$, для которых $z_{k}=(S K)^{k} z$. Криволинейные координаты таких точек обозначим через $\theta_{k}$ и $L_{k}$. Тогда координаты $\left\{A_{k}, B_{k}\right\}$ и $\left\{\theta_{k}, L_{k}\right\}$ связаны равенствами

$$
\left\{\begin{array}{l}
A_{k}=\frac{L_{k}^{4}\left[1-4 \theta_{k}^{3}+2 \theta_{k}^{4}\right]-1}{L_{k}^{3}} \\
B_{k}=\frac{3-L_{k}^{4}\left[1-8 \theta_{k}^{3}+6 \theta_{k}^{4}\right]}{L_{k}^{2}} .
\end{array}\right.
$$

Определение отображения $S K$ дает формулы

$$
\left\{\begin{array}{l}
A_{k+1}=\frac{1-L_{k}^{4}\left[1-4\left(1-\theta_{k}\right)^{3}+2\left(1-\theta_{k}\right)^{4}\right]}{L_{k}^{3}} \\
B_{k+1}=\frac{3-L_{k}^{4}\left[1-8\left(1-\theta_{k}\right)^{3}+6\left(1-\theta_{k}\right)^{4}\right]}{L_{k}^{2}} .
\end{array}\right.
$$

Отсюда получаем, что для произвольных $0 \leq \theta_{k} \leq 1$ и $1 \leq L_{k} \leq \frac{2}{\sqrt[4]{10}}$ справедливо

$$
A_{k+1}-A_{k}=\frac{2}{L_{n}^{3}}-4 L_{k} \theta_{k}\left[1-2 \theta_{k}^{2}+\theta_{k}^{3}\right] \geq \frac{2}{L_{k}^{3}}-\frac{5}{4} L_{k} \geq 0
$$

Заметим, что если $z_{k} \neq T$, то имеет место строгое неравенство $A_{k+1}-A_{k}>0$. Для разности вторых координат справедливо представление $B_{k}-B_{k+1}=2 L_{k}^{2}\left[6 \theta_{k}^{2}-4 \theta_{k}^{3}-1\right]$. Отсюда нетрудно получить, что для произвольного $1 \leq L_{k} \leq \frac{2}{\sqrt[4]{10}}$, если $0 \leq \theta_{k} \leq 1 / 2$, то $B_{k}-B_{k+1} \leq 0$, а если $1 / 2 \leq \theta_{k} \leq 1$, то $B_{k}-B_{k+1} \geq 0$.

Отметим, что если точка $z_{k} \neq T$ принадлежит замкнутому четырехугольнику $V M Q T$, то точка $z_{k+1}$ не принадлежит четырехугольнику $V N P T$. Действительно, в этом случае $A_{k+1}>0$.

Покажем, что не существует точки $z \in V M Q T$ такой, что $z \neq T$ и $z_{n}=(S K)^{n} z \in \bar{G}$ для любых натуральных $n$. Предположим, что такая последовательность найдется. Тогда, как показано выше, все элементы последовательности $z_{n}$ принадлежат $V M Q T$; последовательность $A_{n}$ возрастает, а последовательность $B_{n}$ не убывает. Тогда последовательность точек $z_{n}$ сходится. При этом предельная точка принадлежит $V M Q T$ и вследствие монотонности координат расположена либо выше, либо правее точки $T$. Таким образом, с точкой $T$ не совпадает. 
С другой стороны, преобразование $S K$ является непрерывным. Поэтому предельная точка должна быть неподвижной точкой преобразования $S K$. По лемме 1 такая точка единственная и это точка $T$. Первая часть леммы доказана.

Предположим теперь, что существует точка $z \in V N P T$ такая, что $z_{n}=(S K)^{n} z \in \bar{G}$ для любых натуральных $n$. Тогда из первой части леммы следует, что все элементы последовательности принадлежат четырехугольнику $V N P T$; координаты точек являются монотонными последовательностями: $A_{n}$ возрастает, $B_{n}$ не возрастает. Тогда последовательность сходится, а значит она сходится к точке $T$.

В следующей лемме будет показано существование точки $z \in V N P T$ такой, что $(S K)^{n} z \in$ $\bar{G}$ для любых натуральных $n$. Более того, что такая точка найдется на дуге $M U$.

Рассмотрим два множества $Y_{1}$ и $Y_{2}$ точек кривой $M U$, обладающих следующими свойствами: $Y_{1}$ состоит из точек открытой дуги $M U$ (обозначим их $z$ ) таких, что существует натуральное число $n$ (может быть, зависящее от $z$ ), для которого $(S K)^{n-1} z \in \bar{G}$ и $(S K)^{n} z \notin \bar{G}$, причем $(S K)^{n} z$ принадлежит правой полуплоскости; $Y_{2}$-множество точек $z$ открытой дуги $M U$ таких, что $\exists n \in \mathbb{N}$ (может быть зависящее от $z$ ), для которого $(S K)^{n-1} z \in \bar{G}$, а $(S K)^{n} z \notin \bar{G}$ и $(S K)^{n} z$ принадлежит левой полуплоскости.

Лемма 3. На дуге $M U$, параметрическое уравнение которой имеет вид

$$
\left\{\begin{array}{l}
x=\frac{1-\alpha^{4}}{\alpha^{3}}, \\
y=\frac{3-\alpha^{4}}{\alpha^{2}},
\end{array} \quad \alpha \in\left[1 ; \frac{2}{\sqrt[4]{10}}\right],\right.
$$

найдется по крайней мере одна точка $z_{0}$, обладающая свойством $(S K)^{n} z_{0} \in G$ для любого $n \in \mathbb{N}$.

Д о к а з а т е л ь с т в о. Множества $Y_{1}$ и $Y_{2}$ не пусты, поскольку множество $Y_{1}$ содержит точки дуги $M F$, а $Y_{2}$ состоит из точек дуги $M U$, близких к точке $U$.

Поскольку преобразования $K$ и $S$ являются непрерывными отображениями, то нетрудно понять, что множества $Y_{1}$ и $Y_{2}$ открыты по отношению к дуге $M U$. Так как множества $Y_{1}$ и $Y_{2}$ не пусты и не пересекаются, то они не исчерпываютт всю дугу $M U$. Тогда на дуге $M U$ найдется хотя бы одна точка $z_{0}$, для которой $(S K)^{n} z_{0} \in G$ для всех натуральных $n$.

Обозначим через $\alpha_{0}$ значение параметра $\alpha$, соответствующее точке $z_{0}$. Точка $z_{0}$ будет играть важнейшую роль при построении экстремальной функции $\varphi(t)$. Чтобы вычислить точные значения $\mu_{41}, \mu_{42}, \mu_{43}$, потребуется умение вычислять координаты точки $z_{0}$. С этой целью будет построен соответствующий алгоритм. Пусть $z_{n}=(S K)^{n} z_{0}$, а $\theta_{n}$ и $L_{n}-$ криволинейные координаты точки $z_{n}$.

Лемма 4. Криволинейные координаты $\theta_{n}$ и $L_{n}$ точек $z_{n}$ удовлетворяют равенствам

$$
\theta_{n}=\frac{1}{2}\left(1+\sigma_{n}\right), \quad L_{n}=\frac{2}{\sqrt[4]{10}}\left(1-\tau_{n}\right),
$$

где $\sigma_{n}>0, \tau_{n}>0 u \sum_{n=1}^{\infty} \sigma_{n}<\infty, \sum_{n=1}^{\infty} \tau_{n}<\infty$.

Д о к а з а т е л ь с т в о. В лемме 2 доказано, что предел последовательности $z_{n}$ совпадает с точкой $T$. Отсюда вытекает, что $\sigma_{n} \rightarrow 0$ и $\tau_{n} \rightarrow 0$. Для декартовых координат точки $z_{n+1}$ вместе с формулой (2.3) справедливы формулы

$$
\left\{\begin{array}{l}
A_{n+1}=\frac{L_{n+1}^{4}\left[1-4 \theta_{n+1}^{3}+2 \theta_{n+1}^{4}\right]-1}{L_{n+1}^{3}}, \\
B_{n+1}=\frac{3-L_{n+1}^{4}\left[1-8 \theta_{n+1}^{3}+6 \theta_{n+1}^{4}\right]}{L_{n+1}^{2}} .
\end{array}\right.
$$


Сравнивая эти выражения с выражениями (2.3), получаем систему

$$
\left\{\begin{array}{c}
\frac{1-\frac{8}{5}\left(1-\tau_{n}\right)^{4}\left[1-\frac{1}{2}\left(1-\sigma_{n}\right)^{3}+\frac{1}{8}\left(1-\sigma_{n}\right)^{4}\right]}{\left(1-\tau_{n}\right)^{3}} \\
=\frac{\frac{8}{5}\left(1-\tau_{n+1}\right)^{4}\left[1-\frac{1}{2}\left(1+\sigma_{n+1}\right)^{3}+\frac{1}{8}\left(1+\sigma_{n+1}\right)^{4}\right]-1}{\left(1-\tau_{n+1}\right)^{3}} \\
\frac{3-\frac{8}{5}\left(1-\tau_{n}\right)^{4}\left[1-\left(1-\sigma_{n}\right)^{3}+\frac{3}{8}\left(1-\sigma_{n}\right)^{4}\right]}{\left(1-\tau_{n}\right)^{2}} \\
=\frac{3-\frac{8}{5}\left(1-\tau_{n+1}\right)^{4}\left[1-\left(1+\sigma_{n+1}\right)^{3}+\frac{3}{8}\left(1+\sigma_{n+1}\right)^{4}\right]}{\left(1-\tau_{n+1}\right)^{2}}
\end{array}\right.
$$

Зависимость переменных $\tau_{n+1}, \sigma_{n+1}$ от переменных $\tau_{n}$ и $\sigma_{n}$ выражается системой

$$
\left\{\begin{array}{l}
{\left[5+\varepsilon_{11}^{\prime}(n)\right] \tau_{n+1}+\left[2+\varepsilon_{12}^{\prime}(n)\right] \sigma_{n+1}=\left[-5+\varepsilon_{11}^{\prime \prime}(n)\right] \tau_{n}+\left[2+\varepsilon_{12}^{\prime \prime}(n)\right] \sigma_{n}} \\
{\left[3+\varepsilon_{21}^{\prime}(n)\right] \tau_{n+1}+\left[1+\varepsilon_{22}^{\prime}(n)\right] \sigma_{n+1}=\left[3+\varepsilon_{21}^{\prime \prime}(n)\right] \tau_{n}+\left[-1+\varepsilon_{22}^{\prime \prime}(n)\right] \sigma_{n}}
\end{array}\right.
$$

где $\varepsilon_{\nu \mu}^{\prime}(n) \rightarrow 0, \varepsilon_{\nu \mu}^{\prime \prime}(n) \rightarrow 0$ при $n \rightarrow \infty, \mu, \nu=1,2$. Решая последнюю систему относительно $\tau_{n+1}$ и $\sigma_{n+1}$, приходим к следующим выражениям:

$$
\left\{\begin{array}{l}
\tau_{n+1}=\left[11+\varepsilon_{11}(n)\right] \tau_{n}+\left[-4+\varepsilon_{12}(n)\right] \sigma_{n} \\
\sigma_{n+1}=\left[-30+\varepsilon_{21}(n)\right] \tau_{n}+\left[11+\varepsilon_{22}(n)\right] \sigma_{n}
\end{array}\right.
$$

где $\varepsilon_{\nu \mu}(n) \rightarrow 0$ при $n \rightarrow \infty$, а $\nu, \mu=1,2$.

Завершая доказательство, покажем, что $\sum_{n=1}^{\infty} \tau_{\nu}<\infty$ и $\sum_{n=1}^{\infty} \sigma_{n}<\infty$.

Om противного. Пусть последовательности $\left\{n_{k}\right\}$ и $\left\{m_{k}\right\}$ таковы, что $\sum_{\nu=n_{k}}^{n_{k}+m_{k}} \tau_{\nu} \rightarrow \infty$, если $k \rightarrow \infty$. Суммируя соотношения системы (2.4), получаем

$$
\tau_{n_{k}+m_{k}+1}-\tau_{n_{k}}+\sum_{\nu=n_{k}}^{n_{k}+m_{k}} \tau_{\nu}=11 \sum_{\nu=n_{k}}^{n_{k}+m_{k}} \tau_{\nu}+\sum_{\nu=n_{k}}^{n_{k}+m_{k}} \varepsilon_{11}(\nu) \tau_{\nu}-4 \sum_{\nu=n_{k}}^{n_{k}+m_{k}} \sigma_{\nu}+\sum_{\nu=n_{k}}^{n_{k}+m_{k}} \varepsilon_{12}(\nu) \sigma_{\nu},
$$

откуда

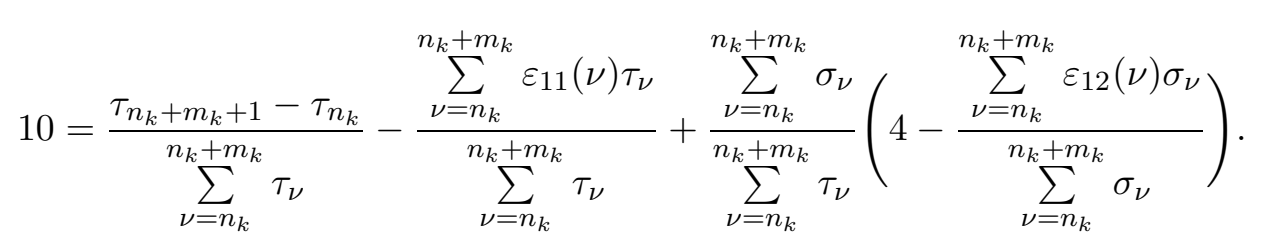

В наших предположениях первые два слагаемые правой части (2.5) при $k \rightarrow \infty$ стремятся к нулю. Отсюда следует, что ряд $\sum_{\nu=1}^{\infty} \sigma_{\nu}$ расходится, т. е. $\sum_{\nu=1}^{\infty} \sigma_{\nu}=\infty$ и поэтому

$$
\frac{\sum_{\nu=n_{k}}^{n_{k}+m_{k}} \sigma_{\nu}}{\sum_{\nu=n_{k}}^{n_{k}+m_{k}} \tau_{\nu}} \rightarrow \frac{5}{2}
$$

Проводя такие же рассуждения для второго равенства системы (2.4), получаем

$$
\frac{\sum_{\nu=n_{k}}^{n_{k}+m_{k}} \sigma_{\nu}}{\sum_{\nu=n_{k}}^{n_{k}+m_{k}} \tau_{\nu}} \rightarrow 3
$$


Установленное противоречие показывает, что $\sum_{\nu=1}^{\infty} \tau_{n}<\infty$, а значит (см. (2.5)),

$$
\sum_{\nu=1}^{\infty} \sigma_{\nu}<\infty
$$

Можно доказать, что следствием леммы является более сильное утверждение.

Следствие 1. Для последовательностей $\tau_{n}$ u $\sigma_{n}$ при $n \rightarrow \infty$ имеем

$$
\frac{\tau_{n+1}}{\tau_{n}} \rightarrow 11-\sqrt{120}, \quad \frac{\sigma_{n+1}}{\sigma_{n}} \rightarrow 11-\sqrt{120} .
$$

\section{3. Функция $\varphi$}

Построим функцию $\varphi$, экстремальную для неравенств (1.2).

Лемма 5. Существует последовательность чисел

$$
0<a<b<x_{1}<\xi_{1}<x_{2}<\xi_{2}<\ldots \quad\left(x_{k} \rightarrow \infty \text { npu } k \rightarrow \infty\right)
$$

и функиия $\varphi$ такие, что

1) $\varphi \in \Omega_{4}[0, \infty)$;

2) $\varphi^{I V}(t)= \begin{cases}(-1)^{n+1} \cdot 48, & t \in\left(x_{n}, x_{n+1}\right), n=\overline{1, \infty}, \\ -48, & t \in\left(0, x_{1}\right) ;\end{cases}$

3) $\left\{\begin{array}{l}\varphi(0)=\varphi(b)=\varphi\left(\xi_{2 k}\right)=-1, \quad k=\overline{1, \infty} \\ \varphi(a)=\varphi\left(\xi_{2 k-1}\right)=+1, \quad k=\overline{1, \infty}\end{array}\right.$

4) если обозначить

$$
\xi_{k}-x_{k}=\frac{1}{\sqrt[4]{10}}+\varkappa_{k}^{\prime} \quad u \quad x_{k}-\xi_{k-1}=\frac{1}{\sqrt[4]{10}}+\varkappa_{k}^{\prime \prime},
$$

mo

$$
\sum_{k=1}^{\infty}\left|\varkappa_{k}^{\prime}\right|<\infty \quad u \sum_{k=1}^{\infty}\left|\varkappa_{k}^{\prime \prime}\right|<\infty
$$

Д о к а з а т е л ь с т в о. В лемме 3 доказано существование точки $z_{0}$ на дуге $M U$. Обозначим $z_{n}=(S K)^{n} z_{0}$ и ее декартовы координаты обозначим $A_{n}$ и $B_{n}$. Имеем

$$
A_{0}=\frac{1-\alpha_{0}^{4}}{\alpha_{0}^{3}}, \quad B_{0}=\frac{3-\alpha_{0}^{4}}{\alpha_{0}^{2}} .
$$

Пусть $\theta_{n}$ и $L_{n}-$ криволинейные координаты точки $z_{n}$. Из определения криволинейных координат вытекает

$$
\left\{\begin{array}{l}
A_{n}=\frac{L_{n}^{4}\left(1-4 \theta_{n}^{3}+2 \theta_{n}^{4}\right)-1}{L_{n}^{3}}, \\
B_{n}=\frac{3-L_{n}^{4}\left(1-8 \theta_{n}^{3}+6 \theta_{n}^{4}\right)}{L_{n}^{2}} .
\end{array}\right.
$$

Построение операторов $K$ и $S$ приводит к следующим соотношениям для координат точки $z_{n+1}=S K z_{n}$

$$
\left\{\begin{array}{l}
A_{n+1}=\frac{1-L_{n}^{4}\left[1-4\left(1-\theta_{n}\right)^{3}+2\left(1-\theta_{n}\right)^{4}\right]}{L_{n}^{3}}, \\
B_{n+1}=\frac{3-L_{n}^{4}\left[1-8\left(1-\theta_{n}\right)^{3}+6\left(1-\theta_{n}\right)^{4}\right]}{L_{n}^{2}} .
\end{array}\right.
$$


Построим алгебраические многочлены для $n \in \mathbb{N}$ вида

$$
p_{n}(t)=(-1)^{n+1}\left[2 t^{4}-4 A_{n} t^{3}-2 B_{n} t^{2}+1\right] .
$$

Определим последовательность (3.1) следующими соотношениями:

$$
\begin{gathered}
a=\frac{\sqrt{1+\alpha_{0}^{4}}-1}{\alpha_{0}^{3}}, \quad b=\frac{\sqrt{1+\alpha_{0}^{4}}+\alpha_{0}^{4}-1}{\alpha_{0}^{3}}, \\
x_{1}-b=L_{0}\left(1-\theta_{0}\right), \quad \xi_{1}-x_{1}=L_{0} \theta_{0}, \\
x_{n}-\xi_{n-1}=L_{n-1}\left(1-\theta_{n-1}\right), \quad n=\overline{2, \infty}, \quad \xi_{n}-x_{n}=L_{n-1} \theta_{n-1}, \quad n=\overline{2, \infty} .
\end{gathered}
$$

Положим

$$
\varphi(t)= \begin{cases}p_{0}(t-b) & \text { для } 0 \leq t<x_{1} \\ p_{n}\left(t-\xi_{n}\right) & \text { для } \quad x_{n} \leq t<x_{n+1}, \quad n=\overline{1, \infty} .\end{cases}
$$

Поскольку $\xi_{n}-\xi_{n-1}=L_{n-1}$, а $L_{n} \rightarrow \frac{2}{\sqrt[4]{10}}$ при $n \rightarrow \infty$ в силу леммы 4 , отсюда получаем, что $\xi_{n} \rightarrow+\infty$ и поэтому $x_{n} \rightarrow+\infty$. Как следует из леммы 4 для последовательности (3.1) выполнен п. 4) доказываемой леммы.

Пункт 2) для функции $\varphi$ выполняется очевидным образом.

Имеем далее, что $\varphi\left(\xi_{n}\right)=p_{n}(0)=(-1)^{n+1}$ при $n=\overline{1, \infty}$ и $\varphi(b)=p_{0}(0)=-1$. Непосредственно проверяется, что

$$
\varphi(0)=p_{0}(-b)=p_{0}\left(-\frac{\sqrt{1+\alpha_{0}^{4}}+\alpha_{0}^{4}-1}{\alpha_{0}^{3}}\right)=-1, \quad \varphi(a)=p_{0}(a-b)=p_{0}\left(-\alpha_{0}\right)=+1 .
$$

Это означает выполнение п. 3) для функции $\varphi(t)$.

Проверим, наконец, выполнение утверждения 1) леммы для функции $\varphi(t)$. Для удобства записи обозначим $b=\xi_{0}$. Для установления непрерывности $\varphi, \varphi^{\prime}, \varphi^{\prime \prime}, \varphi^{\prime \prime \prime}$ проверим равенства

$$
p_{n}^{(j)}\left(x_{n+1}-\xi_{n}\right)=p_{n+1}^{(j)}\left(x_{n+1}-\xi_{n+1}\right) \quad \text { для } j=\overline{0,3} \text { и } n=0,1,2, \ldots
$$

Подстановкой $A_{n}, B_{n}, A_{n+1}$ и $B_{n+1}$ из (3.4) и (3.5) непосредственно проверяется справедливость указанных соотношений.

Для завершения доказательства леммы остается доказать, что $\|\varphi\|=1$. На участке $\left[0, \xi_{0}\right]$ функция $\varphi$ определяется выражением

$$
-2\left(t-\xi_{0}\right)^{4}+4 \frac{1-2 \alpha_{0}^{4}}{\alpha_{0}^{3}}\left(t-\xi_{0}\right)^{3}+2 \frac{3-\alpha_{0}^{4}}{\alpha_{0}^{2}}\left(t-\xi_{0}\right)^{2}-1 .
$$

В точке $\xi_{0}$ эта функция имеет локальный минимум, поскольку $\varphi^{\prime}\left(\xi_{0}\right)=0$ и $\varphi^{\prime \prime}\left(\xi_{0}\right)>0$. Это означает, что на $\left[0, \xi_{0}\right]$ функция $\varphi$ имеет лишь один локальный максимум. Очевидно, что этот локальный максимум имеет место в точке $a$, поскольку $\varphi^{\prime}(a)=0$ и $\varphi^{\prime \prime}(a)<0$ (рис. 4 ).

Из равенств $\varphi(0)=\varphi\left(\xi_{0}\right)=-1$ и $\varphi(a)=1$ в силу того, что на отрезках $[0, a]$ и $\left[a, \xi_{0}\right]$ функция $\varphi(t)$ монотонна, следует $\max _{t \in\left[0, \xi_{0}\right]}|\varphi(t)|=1$.

Рассмотрим функцию $\varphi$ на отрезке $\left[\xi_{2 k}, \xi_{2 k+1}\right]$ (рис. 5,6 ). По определению

$$
\varphi(t)=\left\{\begin{array}{l}
-2\left(t-\xi_{2 k}\right)^{4}+4 A_{2 k}\left(t-\xi_{2 k}\right)^{3}+2 B_{2 k}\left(t-\xi_{2 k}\right)^{2}-1 \text { для } \xi_{2 k}<t<x_{2 k+1} ; \\
2\left(t-\xi_{2 k+1}\right)^{4}-4 A_{2 k+1}\left(t-\xi_{2 k+1}\right)^{3}-2 B_{2 k+1}\left(t-\xi_{2 k+1}\right)^{2}+1 \text { для } x_{2 k+1}<t<\xi_{2 k+1},
\end{array}\right.
$$

где $B_{2 k}>0$ и $B_{2 k+1}>0$. 


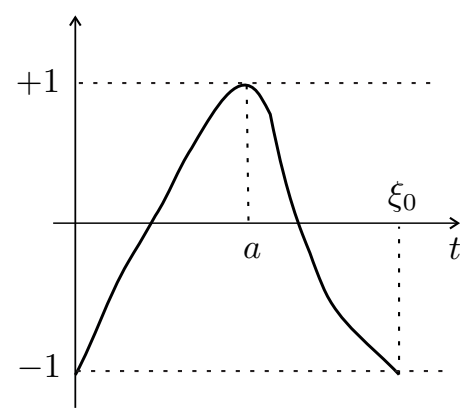

Рис. 4

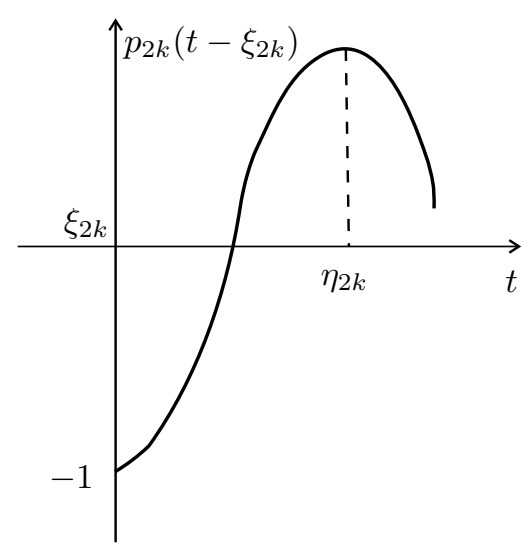

Рис. 5

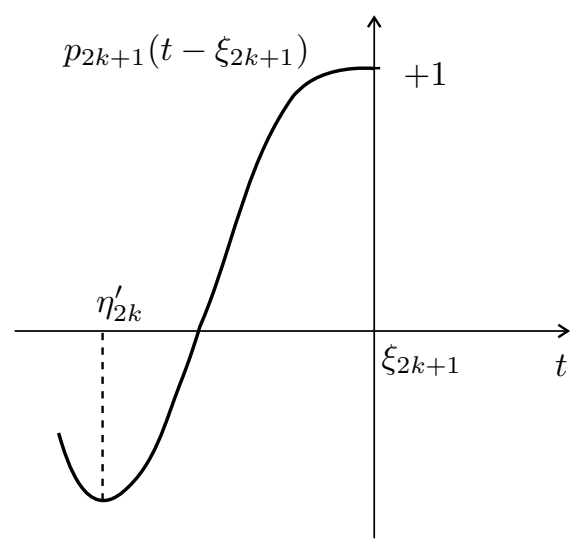

Рис. 6

Многочлен $p_{2 k}\left(t-\xi_{2 k}\right)$ (соответственно $\left.p_{2 k+1}\left(t-\xi_{2 k+1}\right)\right)$ имеет в точке $\xi_{2 k}$ (соответственно в точке $\xi_{2 k+1}$ ) локальный минимум (соответственно локальный максимум). Это означает, что правее (соответственно левее) точки $\xi_{2 k}$ (соответственно точки $\xi_{2 k+1}$ ) имеется один и только один локальный максимум (соответственно минимум) в точке $\eta_{2 k}$ (соответственно $\eta_{2 k}^{\prime}$ ). Точка $x_{2 k+1}$ расположена на интервале $\left(\xi_{2 k}, \xi_{2 k+1}\right)$. Эта точка не может располагаться правее $\eta_{2 k}$, так как в этом случае нарушается либо условие $p_{2 k}^{\prime \prime}\left(x_{2 k+1}-\xi_{2 k}\right)=p_{2 k+1}^{\prime \prime}\left(x_{2 k+1}-\xi_{2 k+1}\right)$, либо условие $p_{2 k}^{\prime}\left(x_{2 k+1}-\xi_{2 k}\right)=p_{2 k+1}^{\prime}\left(x_{2 k+1}-\xi_{2 k+1}\right)$. По той же причине $x_{2 k+1}$ не может лежать левее $\eta_{2 k}^{\prime}$. Это означает, что $x_{2 k+1}<\eta_{2 k}$ и $\eta_{2 k}^{\prime}<x_{2 k+1}$. Тогда $\varphi$ не убывает на $\left[\xi_{2 k}, \xi_{2 k+1}\right]$. Отсюда

$$
\max _{t \in\left[\xi_{2 k}, \xi_{2 k+1}\right]}|\varphi(t)|=1
$$

Аналогичные рассуждения справедливы для $t \in\left[\xi_{2 k-1}, \xi_{2 k}\right]$.

3 а м е ч а н и е. Нетрудно понять, что всякая функция $\varphi$, удовлетворяющая пп. 1) -3 ) леммы, обязательно удовлетворяет п. 4).

\section{4. Двойственные функции $\Phi_{j}, j=\overline{1,3}$}

Для построения функций $\Phi_{j}, j=\overline{1,3}$, будет использована последовательность $(3.1)$, полученная в лемме 5.

Для удобства вновь обозначим $b=\xi_{0}$. Введем новые переменные для всех $\nu=\overline{1, \infty}$

$$
h_{\nu}=x_{\nu}-\xi_{\nu-1}=L_{\nu-1}\left(1-\theta_{\nu-1}\right), \quad H_{\nu}=\xi_{\nu}-x_{\nu}=L_{\nu-1} \theta_{\nu-1} .
$$

В этих обозначениях формулы (3.2) можно записать как

$$
h_{\nu}=\frac{1}{\sqrt[4]{10}}+\varkappa_{\nu}^{\prime} \text { и } H_{\nu}=\frac{1}{\sqrt[4]{10}}+\varkappa_{\nu}^{\prime \prime}
$$

и согласно лемме 5 выполнено (3.3).

Каждому отрезку $\left[\xi_{\nu-1}, \xi_{\nu}\right]$ поставим в соответствие трехмерный вектор $r_{v}=\left\{a_{\nu}, b_{\nu}, c_{\nu}\right\}$. Построим на полуоси $\left[\xi_{0}, \infty\right)$ функцию

$$
\Phi(t)=(-1)^{\nu+1}\left[a_{\nu}\left(t-x_{\nu}\right)+\sqrt[4]{10} b_{\nu}\left(t-x_{\nu}\right)^{2}+\sqrt{10} c_{\nu}\left(t-x_{\nu}\right)^{3}\right], \text { если } t \in\left[\xi_{\nu-1}, \xi_{\nu}\right] .
$$

Выясним условия, при каких функция $\Phi$ будет непрерывной на $\left[\xi_{0}, \infty\right)$ вместе со своими производными первого и второго порядка. Эти условия в точке $\xi_{\nu}$ (рис. 7 ) имеют вид

$$
\left\{\begin{array}{l}
a_{\nu} H_{\nu}+\sqrt[4]{10} b_{\nu} H_{\nu}^{2}+\sqrt{10} c_{\nu} H_{\nu}^{2}=a_{\nu+1} h_{\nu+1}-\sqrt[4]{10} b_{\nu+1} h_{\nu+1}^{2}+\sqrt{10} c_{\nu} h_{\nu+1}^{3} \\
a_{\nu}+2 \sqrt[4]{10} b_{\nu} H_{\nu}+3 \sqrt{10} c_{\nu} H_{\nu}^{2}=-a_{\nu+1}+2 \sqrt[4]{10} b_{\nu+1} h_{\nu+1}-3 \sqrt{10} c_{\nu+1} h_{\nu+1}^{2} \\
2 \sqrt[4]{10} b_{\nu}+6 \sqrt{10} c_{\nu} H_{\nu}=-2 \sqrt[4]{10} b_{\nu+1}+6 \sqrt{10} c_{\nu+1} h_{\nu+1}
\end{array}\right.
$$




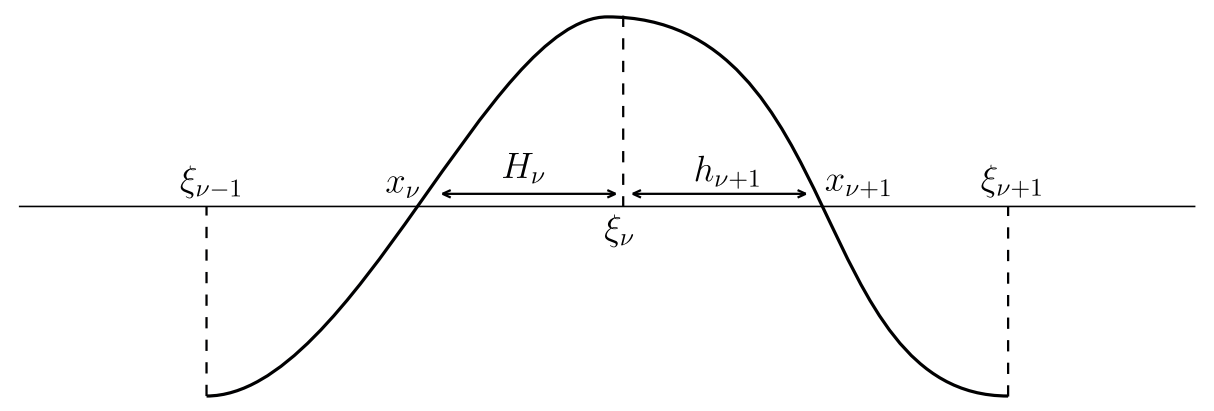

Рис. 7

Отсюда получаем

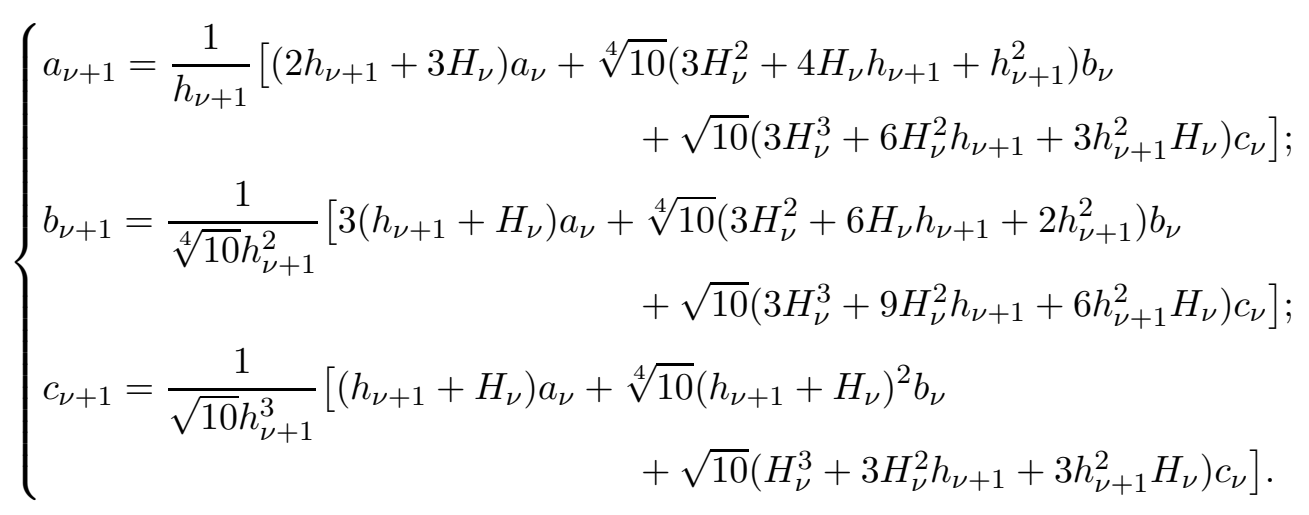

В этом случае обратное преобразование имеет вид

$$
\left\{\begin{aligned}
& a_{\nu}=\frac{1}{H_{\nu}}\left[\left(3 h_{\nu+1}+2 H_{\nu}\right) a_{\nu+1}-\sqrt[4]{10}\right.\left(3 h_{\nu+1}^{2}+4 H_{\nu} h_{\nu+1}+H_{\nu}^{2}\right) b_{\nu+1} \\
&\left.+\sqrt{10}\left(3 h_{\nu+1}^{3}+6 h_{\nu+1}^{2} H_{\nu}+3 h_{\nu+1} H_{\nu}^{2}\right) c_{\nu+1}\right] \\
& b_{\nu}=\frac{1}{\sqrt[4]{10} H_{\nu}^{2}}\left[-3\left(h_{\nu+1}+H_{\nu}\right) a_{\nu+1}\right. \sqrt[4]{10}\left(3 h_{\nu+1}^{2}+6 H_{\nu} h_{\nu+1}+2 H_{\nu}^{2}\right) b_{\nu+1} \\
&\left.-\sqrt{10}\left(3 h_{\nu+1}^{3}+9 h_{\nu+1}^{2} H_{\nu}+6 h_{\nu+1} H_{\nu}^{2}\right) c_{\nu+1}\right] \\
& c_{\nu}=\frac{1}{\sqrt{10} H_{\nu}^{3}}\left[\left(h_{\nu+1}+H_{\nu}\right) a_{\nu+1}-\sqrt[4]{10}\left(h_{\nu+1}^{2}+2 h_{\nu+1} H_{\nu}+H_{\nu}^{2}\right) b_{\nu+1}\right. \\
&\left.+\sqrt{10}\left(h_{\nu}^{3}+3 h_{\nu+1}^{2} H_{\nu}+3 h_{\nu+1} H_{\nu}^{2}\right) c_{\nu+1}\right]
\end{aligned}\right.
$$

Системы (4.2) и (4.3) можно записать в векторной форме $r_{\nu}=P_{\nu} r_{\nu+1}$ и, обратно, $r_{\nu+1}=P_{\nu}^{-1} r_{\nu}$, где $P_{\nu}$ - матрица системы (4.3). Согласно п. 4 леммы 5 и введенным выше обозначениям (4.1)

$$
h_{\nu}=\frac{1}{\sqrt[4]{10}}+\varkappa_{\nu}^{\prime}, \quad H_{\nu}=\frac{1}{\sqrt[4]{10}}+\varkappa_{\nu}^{\prime \prime}, \quad \text { где } \quad \sum_{\nu=1}^{\infty}\left|\varkappa_{\nu}^{\prime}\right|<\infty, \quad \sum_{\nu=1}^{\infty}\left|\varkappa_{\nu}^{\prime \prime}\right|<\infty .
$$

Это значит, что матрице $P_{\nu}$ можно придать форму

$$
P_{\nu}=\left(\begin{array}{ccc}
5+\omega_{11}(\nu) & -8+\omega_{12}(\nu) & 12+\omega_{13}(\nu) \\
-6+\omega_{21}(\nu) & 11+\omega_{22}(\nu) & -18+\omega_{23}(\nu) \\
2+\omega_{31}(\nu) & -4+\omega_{32}(\nu) & 7+\omega_{33}(\nu)
\end{array}\right)
$$

где $\omega_{i j}(\nu)$ таковы, что $\sum_{\nu=1}^{\infty}\left|\omega_{i j}(\nu)\right|<\infty$ для $j, i=1,2,3$.

Пусть $P_{\nu}=P+\Omega_{\nu}$, где $P=\left(\begin{array}{ccc}5 & -8 & 12 \\ -6 & 11 & -18 \\ 2 & -4 & 7\end{array}\right), \quad \sum_{\nu=1}^{\infty}\left\|\Omega_{\nu}\right\|<\infty$ (через $\left\|\Omega_{\nu}\right\|$ обозначена норма линейного оператора, матрицей которого является $\left.\Omega_{\nu}\right)$. 
Нетрудно найти собственные числа матрицы $P$. Они соответственно равны $11-\sqrt{120}, 1$ и $11+\sqrt{120}$ и являются корнями характеристического уравнения $\operatorname{det}(P-\lambda E)=-\lambda^{3}+23 \lambda^{2}-$ $23 \lambda+1=-(\lambda-1)\left(\lambda^{2}-22 \lambda+1\right)$. Положим $\lambda_{0}=11+\sqrt{120}$. Собственный вектор $e$, который соответствует $\lambda_{0}$, имеет координаты $\left\{2,-3 \frac{\lambda_{0}-1}{\lambda_{0}+1}, 1\right\}$.

Обычными методами теории возмущений при достаточно больших $\nu$ можно получить оценку $\lambda_{\nu}=\lambda_{0}\left(1+\rho_{\nu}\right)$, где $\sum_{\nu=1}^{\infty}\left|\rho_{\nu}\right|<\infty$. Откуда при всех $\nu=\overline{1, \infty}$ вытекает неравенство $\left\|P_{\nu}\right\| \leq \lambda_{0}\left(1+\left|\rho_{\nu}\right|\right)$, где $\sum_{\nu=1}^{\infty}\left|\rho_{\nu}\right|<\infty$ (через $\lambda_{\nu}$ обозначено максимальное собственное значение матрицы $\left.P_{\nu}\right)$.

Лемма 6. Пусть е - собственный вектор матрицы $P$, соответствующий собственному значению $\lambda_{0}=11+\sqrt{120}$. Тогда существует вектор $e_{1}=\lim _{N \rightarrow \infty} \frac{1}{\lambda_{0}^{N}} P_{1} P_{2} \cdots P_{N}$ е такой, что векторы е $e_{s+1}=P_{s}^{-1} P_{s-1}^{-1} \cdots P_{1}^{-1} e_{1}$ обладают следуюшими свойствами:

1) При всех натуральных s первые и третъи координаты векторов е вторые координаты отрицательны;

2) $\left\|e_{s}\right\|=\underline{\underline{O}}\left(\frac{1}{\lambda_{0}^{s}}\right)$.

Д о к а з а т е л ь с т в о. Сначала покажем существование ненулевого вектора

$$
e_{1}=\lim _{N \rightarrow \infty} \frac{1}{\lambda_{0}^{N}} P_{1} P_{2} \cdots P_{N} e
$$

Имеем

$$
\begin{gathered}
\left\|\frac{1}{\lambda_{0}^{n+p}} P_{1} P_{2} \cdots P_{n+p} e-\frac{1}{\lambda_{0}^{n}} P_{1} P_{2} \cdots P_{n} e\right\| \leq \frac{1}{\lambda_{0}^{n}}\left\|P_{1}\right\| \cdot\left\|P_{2}\right\| \cdots\left\|P_{n}\right\| \cdot\left\|\frac{1}{\lambda_{0}^{p}} P_{n+1} P_{n+2} \cdots P_{n+p} e-e\right\| \\
\leq \prod_{\nu=1}^{n}\left(1+\left|\rho_{\nu}\right|\right)\left\|\frac{1}{\lambda_{0}^{p}} P_{n+1} \cdots P_{n+p} e-e\right\| \leq C_{1}\left\|\prod_{s=n+1}^{n+p}\left(P+\Omega_{s}\right) e-e\right\|,
\end{gathered}
$$

здесь $C_{1}$ не зависит от $n$ и от $p$. Отсюда следует, что правая часть последнего неравенства удовлетворяет неравенству

$$
\begin{gathered}
\left\|\frac{1}{\lambda_{0}^{p}} P_{n+1} P_{n+2} \cdots P_{n+p} e-e\right\|=\left\|\frac{1}{\lambda_{0}} P_{n+1}\left(\frac{1}{\lambda_{0}^{p-1}} \prod_{s=n+2}^{n+p} P_{s} e-e\right)+\frac{1}{\lambda_{0}} P_{n+1} e-e\right\| \\
\leq\left[\frac{1}{\lambda_{0}}\left\|P+\Omega_{n+1}\right\| \cdot\left\|\frac{1}{\lambda_{0}^{p-1}} \prod_{s=n+2}^{n+p}\left(P+\Omega_{s}\right) e-e\right\|+\frac{1}{\lambda_{0}}\left\|P_{n+1} e-\lambda_{0} e\right\|\right] \\
\leq\left[\left(1+\rho_{n+1}\right)\left\|\frac{1}{\lambda_{0}^{p-1}} \prod_{s=n+2}^{n+p}\left(P+\Omega_{s}\right) e-e\right\|+\frac{1}{\lambda_{0}}\left\|\Omega_{n+1} e\right\|\right] .
\end{gathered}
$$

Для разности

$$
\left\|\frac{1}{\lambda_{0}^{p-1}} \prod_{s=n+2}^{n+p}\left(P+\Omega_{s}\right) e-e\right\|=\left\|\frac{1}{\lambda_{0}} P_{n+2}\left(\frac{1}{\lambda_{0}^{p-2}} \prod_{s=n+3}^{n+p}\left(P+\Omega_{s}\right) e-e\right)+\frac{1}{\lambda_{0}} P_{n+2} e-e\right\|
$$

справедлива оценка, аналогичная той, что получена выше, а именно

$$
\left\|\frac{1}{\lambda_{0}^{p-1}} \prod_{s=n+2}^{n+p}\left(P+\Omega_{s}\right) e-e\right\| \leq\left[\frac{1}{\lambda_{0}}\left\|\Omega_{n+2} e\right\|+\left(1+\rho_{n+2}\right)\left\|\frac{1}{\lambda_{0}^{p-2}} \prod_{s=n+3}^{n+p}\left(P+\Omega_{s}\right) e-e\right\|\right] \text { и т. д. }
$$


Окончательно получаем

$$
\begin{gathered}
\left\|\frac{1}{\lambda_{0}^{p}} P_{n+1} \cdots P_{n+p} e-e\right\| \leq \cdots \\
\leq\left[\frac{1}{\lambda_{0}}\left\|\Omega_{n+1} e\right\|+\frac{\left(1+\rho_{n+1}\right)}{\lambda_{0}}\left\|\Omega_{n+2} e\right\|+\cdots+\frac{\left(1+\rho_{n+1}\right) \cdots\left(1+\rho_{n+p-1}\right)}{\lambda_{0}}\left\|\Omega_{n+p} e\right\|\right. \\
\left.+\left(1+\rho_{n+1}\right) \cdots\left(1+\rho_{n+p-1}\right)\left\|\frac{1}{\lambda_{0}}\left(P+\Omega_{n+p}\right) e-e\right\|\right] \\
\leq \frac{1}{\lambda_{0}}\left[\left\|\Omega_{n+1}\right\|+\left(1+\rho_{n+1}\right)\left\|\Omega_{n+2}\right\|+\cdots+\left(1+\rho_{n+1}\right) \cdots\left(1+\rho_{n+p-1}\right)\left\|\Omega_{n+p}\right\|\right]\|e\| \\
\leq C_{2}\left[\left\|\Omega_{n+1}\right\|+\left\|\Omega_{n+2}\right\|+\cdots+\left\|\Omega_{n+p}\right\|\right]<\varepsilon,
\end{gathered}
$$

где $C_{2}$ не зависит от $n$ и $p$. Оценка справедлива при всех $p$ и всех $n \geq N(\varepsilon)$. Отсюда имеем, что существует вектор $e_{1}=\lim _{N \rightarrow \infty} \frac{1}{\lambda_{0}^{N}} P_{1} P_{2} \cdots P_{N} e$. Убедимся, что $e_{1} \neq 0$. Из полученной оценки следует, что

$$
\left\|\frac{1}{\lambda_{0}^{p}} P_{n+1} P_{n+2} \cdots P_{n+p} e-e\right\| \leq C_{2} \sum_{s=n+1}^{\infty}\left\|\Omega_{s}\right\| .
$$

Поскольку она верна для всех натуральных $p$, то можно указать такое $n$ при всех $p=\overline{1, \infty}$, что будет справедливым следующее неравенство: $\left\|\frac{1}{\lambda_{0}^{p}} P_{n+1} P_{n+2} \cdots P_{n+p} e\right\| \geq \frac{1}{2}\|e\|$. Пусть такое $n$ зафиксировано. Тогда

$$
\begin{gathered}
\frac{1}{2}\|e\| \leq \lambda_{0}^{n}\left\|P_{1}^{-1}\right\| \cdot\left\|P_{2}^{-1}\right\| \cdots\left\|P_{n}^{-1}\right\| \cdot\left\|\frac{1}{\lambda_{0}^{n+p}} P_{1} P_{2} \cdots P_{n+p} e\right\| \\
\leq \lambda_{0}^{n}\left\|P_{1}^{-1}\right\| \cdot\left\|P_{2}^{-1}\right\| \cdots\left\|P_{n}^{-1}\right\|\left[\left\|e_{1}\right\|+\left\|\frac{1}{\lambda_{0}^{p+n}} P_{1} P_{2} \cdots P_{n+p} e-e_{1}\right\|\right] .
\end{gathered}
$$

Устремив $p$ к $+\infty$, получаем, что $\left\|e_{1}\right\| \geq C\|e\|$, где $C>0$. Это означает, что $e_{1} \neq 0$. Из доказанного вытекает, что $e_{1}=\frac{1}{\lambda_{0}^{N}} P_{1} P_{2} \cdots P_{N} e+q_{N}$, где $q_{N} \rightarrow 0$. Отсюда следует

$$
P_{s}^{-1} P_{s-1}^{-1} \cdots P_{1}^{-1} e_{1}=\frac{1}{\lambda_{0}^{N}} P_{s+1} \cdots P_{N} e+P_{s}^{-1} P_{s-1}^{-1} \cdots P_{1}^{-1} q_{N}
$$

Повторяя рассуждения аналогичные приведенным выше, имеем

$$
\begin{gathered}
\left\|P_{s}^{-1} P_{s-1}^{-1} \cdots P_{1}^{-1} e_{1}\right\| \leq \frac{1}{\lambda_{0}^{N}}\left\|P_{s+1}\right\| \cdots\left\|P_{N}\right\| \cdot\|e\|+\left\|P_{s}^{-1}\right\| \cdots\left\|P_{1}^{-1}\right\| \cdot\left\|q_{N}\right\| \\
\leq\left\|P_{s}^{-1}\right\| \cdots\left\|P_{1}^{-1}\right\| \cdot\left\|q_{N}\right\|+\frac{1}{\lambda_{0}^{s}}\left(1+\rho_{s+1}\right) \cdots\left(1+\rho_{N}\right)\|e\|, \quad s<N .
\end{gathered}
$$

Устремляя $N \rightarrow+\infty$, получим $\left\|P_{s}^{-1} P_{s-1}^{-1} \cdots P_{1}^{-1} e_{1}\right\| \leq \frac{K}{\lambda_{0}^{s}}\|e\|$, где $K$ не зависит от $s$. Отсюда следует выполнение утверждения 2) леммы. И, наконец,

$$
\begin{aligned}
\left\|P_{s}^{-1} P_{s-1}^{-1} \cdots P_{1}^{-1} e_{1}-\frac{1}{\lambda_{0}^{s}} e\right\| & \leq\left\|P_{s}^{-1}\right\| \cdot\left\|P_{s-1}^{-1}\right\| \cdots\left\|P_{1}^{-1}\right\| \cdot\left\|q_{N}\right\|+\frac{1}{\lambda_{0}^{s}}\left\|\frac{1}{\lambda_{0}^{N-s}} P_{s+1} \cdots P_{N} e-e\right\| \\
& \leq\left\|P_{s}^{-1}\right\| \cdots\left\|P_{1}^{-1}\right\| \cdot\left\|q_{N}\right\|+\frac{K_{1}}{\lambda_{0}^{s}} \sum_{\nu=s+1}^{\infty}\left\|\Omega_{\nu}\right\|,
\end{aligned}
$$


а поэтому при $N \rightarrow \infty\left\|\lambda_{0}^{s} P_{s}^{-1} \cdots P_{1}^{-1} e_{1}-e\right\| \leq K_{1} \sum_{\nu=s+1}^{\infty}\left\|\Omega_{\nu}\right\|$. Это означает, что при всех больших $s$ знаки координат векторов $e_{s+1}=P_{s}^{-1} \cdots P_{1}^{-1} e_{1}$ совпадают со знаками соответствующих координат вектора

$$
e=\left\{2,-3 \frac{\lambda_{0}-1}{\lambda_{0}+1}, 1\right\}
$$

Из соотношения $e_{s}=P_{s} e_{s+1}$ и (4.3) следует, что первая и третья координаты вектора $e_{s+1}$ положительны, а вторая отрицательна; тогда это имеет место и для координат вектора $e_{s}$.

Лемма 7. Существует функиия $\Phi$, непрерывная на $[b, \infty)$ вместе с производными первого и второго порядка и обладающая следующими свойствами.

1. $\Phi$ является многочленом 3-го порядка на каждом из отрезков $\left[b, \xi_{1}\right],\left[\xi_{1}, \xi_{2}\right], \ldots$ Знаки $\Phi^{\prime \prime \prime}$ на этих отрезках чередуютсл, причем $\Phi^{\prime \prime \prime}(t)>0$ на $\left[b, \xi_{1}\right]$.

2. $\Phi$ сохраняет знак на каждом из интервалов $\left(b, x_{1}\right),\left(x_{1}, x_{2}\right), \ldots$ Знаки $\Phi$ на этих интервалах чередуются, причем $\Phi(t)<0$ на $\left(b, x_{1}\right)$.

3. $\Phi^{\prime}(b)>0, \Phi^{\prime \prime}(b)<0$.

4. $\left|\Phi^{(j)}(t)\right|=\underline{\underline{O}}(\exp \{-k t\}), k>0$ npu $t \rightarrow \infty \partial \Omega я j=\overline{0,3}$.

Д о к а з а т е л ь с т в о. Определим для $t \in\left[\xi_{\nu-1}, \xi_{\nu}\right]$ функцию

$$
\Phi(t)=(-1)^{\nu+1}\left[a_{\nu}\left(t-x_{\nu}\right)+\sqrt[4]{10} b_{\nu}\left(t-x_{\nu}\right)^{2}+\sqrt{10} c_{\nu}\left(t-x_{\nu}\right)^{3}\right]=q_{\nu}\left(t-x_{\nu}\right),
$$

где $a_{\nu}, b_{\nu}, c_{\nu}$ - координаты вектора $e_{\nu}$, построенного в лемме 6 . Поскольку $e_{\nu}=P_{\nu} e_{\nu+1}$, то очевидно, что функция $\Phi$ непрерывна на отрезке $[b, \infty)$ вместе со своими производными первого и второго порядка. По доказанному $c_{\nu}$ - положительные коэффициенты, а, следовательно, утверждение 1 леммы выполнено, т. е. $\Phi^{\prime \prime \prime}(t)>0$ на $\left[b, \xi_{1}\right]$ и на каждом из отрезков $\left[\xi_{1}, \xi_{2}\right], \ldots$ знаки $\Phi^{\prime \prime \prime}$ чередуются.

Рассмотрим отрезок $\left[x_{2 k-1}, x_{2 k}\right]$ и на нем функцию

$$
\Phi(t)=\left\{\begin{array}{l}
a_{2 k-1}\left(t-x_{2 k-1}\right)+\sqrt[4]{10} b_{2 k-1}\left(t-x_{2 k-1}\right)^{2}+\sqrt{10} c_{2 k-1}\left(t-x_{2 k-1}\right)^{3}, t \in\left[x_{2 k-1}, \xi_{2 k-1}\right] \\
-\left[a_{2 k}\left(t-x_{2 k}\right)+\sqrt[4]{10} b_{2 k}\left(t-x_{2 k}\right)^{2}+\sqrt{10} c_{2 k}\left(t-x_{2 k}\right)^{3}\right], t \in\left[\xi_{2 k-1}, x_{2 k}\right] .
\end{array}\right.
$$

На рис. 8 и 9 изображены графики многочленов $q_{2 k-1}\left(t-x_{2 k-1}\right)$ и $q_{2 k}\left(t-x_{2 k}\right)$. Поскольку $\Phi$ непрерывна в точке $x_{2 k-1}$, то она положительна на промежутке $\left(x_{2 k-1}, x_{2 k}\right)$. Из аналогичных соображений вытекает отрицательность $\Phi$ на множестве $\left(x_{2 k}, x_{2 k+1}\right)$.

Неравенства $\Phi(b)<0, \Phi^{\prime}(b)>0, \Phi^{\prime \prime}(b)<0$ очевидны. Это означает, что выполнены пп. 2 и 3 леммы. В лемме 6 было доказано, что $\left\|e_{s}\right\|=\underline{\underline{O}}\left(\frac{1}{\lambda_{0}^{s}}\right)$. Это соотношение обеспечивает выполнение п. 4 леммы, а именно, $\Phi(t), \Phi^{\prime}(t), \Phi^{\prime \prime}(t), \Phi^{\prime \prime \prime}(t) \rightarrow 0$ при $t \rightarrow \infty$. Лемма доказана.

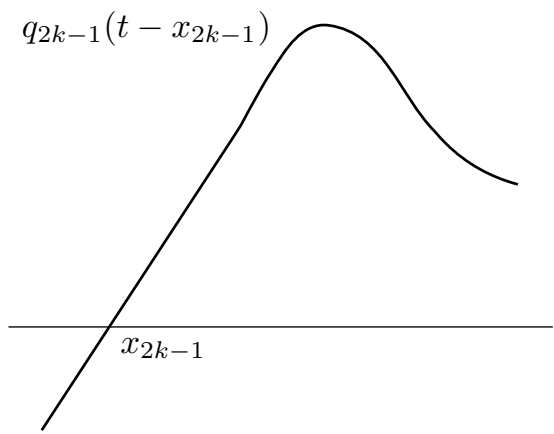

Рис. 8

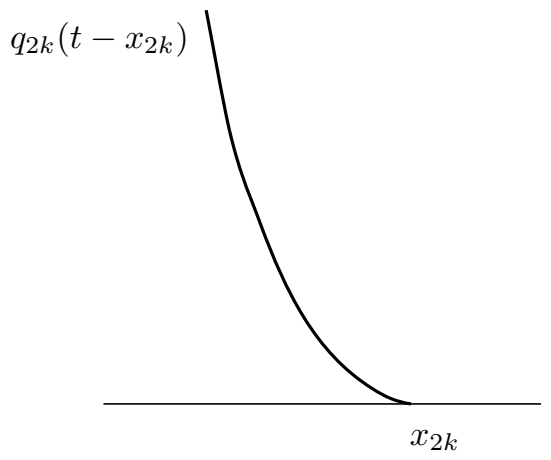

Рис. 9 
Вернемся к последовательности (3.1), по которой построим функции $\Phi_{j}(t), j=\overline{1,3}$, с нужными свойствами.

Лемма 8. Существуют функиии $\Phi_{j}, j=\overline{1,3}$, непрерывные на $[0, \infty)$ вместе с производными первого и второго порядка и обладающие следуюшими свойствами.

1. $\Phi_{j}$ являются алгебраическими многочленами третвей степени на каждом их отрезков $[0, a],[a, b],\left[b, \xi_{1}\right],\left[\xi_{1}, \xi_{2}\right], \ldots$ Знаки $\Phi_{j}^{\prime \prime \prime}$ на этих промежутках чередуются и $\Phi_{j}^{\prime \prime \prime}>0$ на отрезке $[0, a]$.

2. $\Phi_{j}$ сохраняют знаки на каждом из интервалов $\left(0, x_{1}\right),\left(x_{1}, x_{2}\right),\left(x_{2}, x_{3}\right), \ldots$ Знаки $\Phi_{j}$ на этих интервалах чередуются, $a \Phi_{j}(t)<0$ на $\left(0, x_{1}\right)$.

3. $\left|\Phi_{j}^{(s)}(t)\right|=\underline{\underline{O}}(\exp \{-k t\})$ npu $t \rightarrow \infty(k>0) \partial \Omega \Omega j=\overline{1,3}, s=\overline{0,3}$.

4. $\Phi_{1}(0)=\Phi_{1}^{\prime}(0)=0, \Phi_{1}^{\prime \prime}(0)=-1$.

5. $\Phi_{2}(0)=\Phi_{2}^{\prime \prime}(0)=0, \Phi_{2}^{\prime}(0)=-1$.

6. $\Phi_{3}^{\prime}(0)=\Phi_{3}^{\prime \prime}(0)=0, \Phi_{3}(0)=-1$.

Д о к а з а т е л ь с т в о. $\quad$ 1. Построим функцию $\Phi_{1}$. Пусть

$$
\psi_{1}(t)=\left\{\begin{array}{l}
A t^{2}+B t^{3} \text { при } t \in[0, a), \\
A t^{2}+B t^{3}+C(t-a)^{3} \text { при } t \in[a, b), \\
\Phi(t) \text { при } t \in[b, \infty),
\end{array}\right.
$$

где $\Phi(t)$ построена в лемме 7 , а величины $A, B$ и $C$ найдем из системы

$$
\left\{\begin{array}{l}
A b^{2}+B b^{3}+C(b-a)^{3}=\Phi(b) \\
2 A b+3 B b^{2}+3 C(b-a)^{2}=\Phi^{\prime}(b) \\
2 A+6 B b+3 C(b-a)=\Phi^{\prime \prime}(b)
\end{array}\right.
$$

Из построения вытекает, что $\psi_{1}(t)$ непрерывна на $[0, \infty)$ вместе с первой и второй производными. Определитель системы (4.4) имеет вид $\Delta=6 a^{2} b^{2}(b-a)>0$.

Выражения для величин $A, B$ и $C$ имеют вид

$$
\begin{aligned}
& A=\frac{b(b-a)}{\Delta}\left[18 a \Phi(b)-6 a(2 b-a) \Phi^{\prime}(b)+3 a b(b-a) \Phi^{\prime \prime}(b)\right]<0, \\
& B=\frac{b-a}{\Delta}\left[-6(a+b) \Phi(b)+2\left(2 b^{2}+2 a b-a^{2}\right) \Phi^{\prime}(b)-b(b-a)(b+2 a) \Phi^{\prime \prime}(b)\right]>0, \\
& C=\frac{1}{\Delta}\left[6 b^{2} \Phi(b)-4 b^{3} \Phi^{\prime}(b)+b^{4} \Phi^{\prime \prime}(b)\right]<0 .
\end{aligned}
$$

Найдем знак суммы $B+C$ :

$$
B+C=\frac{1}{\Delta}\left[6 a^{2} \Phi(b)-2 a^{2}(3 b-a) \Phi^{\prime}(b)+a^{2} b(3 b-2 a) \Phi^{\prime \prime}(b)\right]<0 .
$$

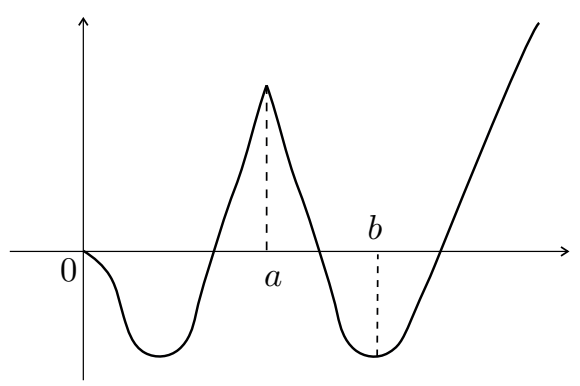

Рис. 10 
Ясно, что пп. 1 и 3 для функции $\psi_{1}(t)$ выполняются. Для того чтобы установить справедливость утверждения 2 леммы, проверим выполнение неравенства $\psi_{1}(t)<0$ на $[0, b]$. Если бы на отрезке $[0, b]$ у функции $\psi_{1}(t)$ менялся знак, то $\psi_{1}(a)>0$, что не может быть в силу второго из равенств системы (4.4) (см. рис. 10) из построения $\psi_{1}(0)=\psi_{1}^{\prime}(0)=0$ и $\psi_{1}^{\prime \prime}(0)=2 A<0$. Функцию $\Phi_{1}(t)$ может быть определена следующим образом:

$$
\Phi_{1}(t)=-\frac{1}{2 A} \psi_{1}(t)
$$

2. Построим функцию $\Phi_{2}$. Рассмотрим

$$
\psi_{2}(t)=\left\{\begin{array}{l}
A t+B t^{3} \text { при } t \in[0, a), \\
A t+B t^{3}+C(t-a)^{3} \text { при } t \in[a, b), \\
\Phi(t) \text { при } t \in[b, \infty),
\end{array}\right.
$$

где $A, B$ и $C$ определяем из системы

$$
\left\{\begin{array}{l}
A b+B b^{3}+C(b-a)^{3}=\Phi(b), \\
A+3 B b^{2}+3 C(b-a)^{2}=\Phi^{\prime}(b), \\
6 B b+6 C(b-a)=\Phi^{\prime \prime}(b) .
\end{array}\right.
$$

По построению $\psi_{2}(t)$ непрерывна на $[0, \infty)$ с производными первого и второго порядка. Определитель системы (4.5) имеет вид $\Delta=6 a b\left(b^{2}-a^{2}\right)>0$. Получим

$$
\begin{aligned}
A & =\frac{1}{\Delta}\left[18 a b(b-a) \Phi(b)-6 a b(b-a)(2 b-a) \Phi^{\prime}(b)+3 a b(b-a)^{2} \Phi^{\prime \prime}(b)\right]<0, \\
B & =\frac{1}{\Delta}\left[-6(b-a) \Phi(b)+6 b(b-a) \Phi^{\prime}(b)-(b-a)^{2}(2 b+a) \Phi^{\prime \prime}(b)\right]>0, \\
C & =\frac{1}{\Delta}\left[6 b \Phi(b)-6 b^{2} \Phi^{\prime}(b)+2 b^{3} \Phi^{\prime \prime}(b)\right]<0, \\
B+C & =\frac{1}{\Delta}\left[6 a \Phi(b)-6 a b \Phi^{\prime}(b)+a\left(3 b^{2}-a^{2}\right) \Phi^{\prime \prime}(b)\right]<0 .
\end{aligned}
$$

Отсюда вытекает, что $\psi_{2}(t)$ не меняет знак на $[0, b]$. Функцию $\Phi_{2}(t)$ определим так:

$$
\Phi_{2}(t)=-\frac{1}{A} \psi_{2}(t) .
$$

3. Построим функцию $\Phi_{3}$. Пусть теперь

$$
\psi_{3}(t)=\left\{\begin{array}{l}
A+B t^{3} \text { при } t \in[0, a), \\
A+B t^{3}+C(t-a)^{3} \text { при } t \in[a, b), \\
\Phi(t) \text { при } t \in[b, \infty) .
\end{array}\right.
$$

Определим $A, B$ и $C$ из системы

$$
\left\{\begin{array}{l}
A+B b^{3}+C(b-a)^{3}=\Phi(b), \\
3 B b^{2}+3 C(b-a)^{2}=\Phi^{\prime}(b) \\
6 B b+6 C(b-a)=\Phi^{\prime \prime}(b)
\end{array}\right.
$$

Отсюда находим $\Delta=18 a b(b-a)$. Имеем

$$
\begin{aligned}
A & =\frac{1}{\Delta}\left[18 a b(b-a) \Phi(b)-6 a b(b-a)(2 b-a) \Phi^{\prime}(b)+3 a b^{2}(b-a)^{2} \Phi^{\prime \prime}(b)\right]<0, \\
B & =\frac{1}{\Delta}\left[6(b-a) \Phi^{\prime}(b)-3(b-a)^{2} \Phi^{\prime \prime}(b)\right]>0, \\
C & =\frac{1}{\Delta}\left[-6 b \Phi^{\prime}(b)+3 b^{2} \Phi^{\prime \prime}(b)\right]<0, \\
B+C & =\frac{1}{\Delta}\left[-6 a \Phi^{\prime}(b)+a(6 b-a) \Phi^{\prime \prime}(b)\right]<0 .
\end{aligned}
$$


Функцию $\Phi_{3}(t)$ определим так:

$$
\Phi_{3}(t)=-\frac{1}{A} \psi_{3}(t)
$$

$\Phi_{3}(t)$ удовлетворяет всем свойствам леммы.

\section{5. Основная теорема}

Рассмотрим произвольную функцию $F \in \Omega_{4}[0, \infty)$. Пусть функция $\Phi_{j}-$ одна из функций леммы $8, j=\overline{1,3}$. Вычислим

$$
\int_{0}^{\infty} F^{I V} \Phi_{j} d t=-F^{\prime \prime \prime}(0) \Phi_{j}(0)+F^{\prime \prime}(0) \Phi_{j}^{\prime}(0)-F^{\prime}(0) \Phi_{j}^{\prime \prime}(0)+F(0) \Phi_{j}^{\prime \prime \prime}(0)+\int_{0}^{\infty} F d \Phi_{j}^{\prime \prime \prime}(t) .
$$

Отсюда следует

$$
\begin{gathered}
-F^{\prime}(0) \Phi_{j}^{\prime \prime}(0)+F^{\prime \prime}(0) \Phi_{j}^{\prime}(0)-F^{\prime \prime \prime}(0) \Phi_{j}(0)=-F(0) \Phi_{j}^{\prime \prime \prime}(0)+F(a)\left[\Phi_{j}^{\prime \prime \prime}(a-0)-\Phi_{j}^{\prime \prime \prime}(a+0)\right] \\
+\sum_{\nu=0}^{\infty} F\left(\xi_{\nu}\right)\left[\Phi_{j}^{\prime \prime \prime}\left(\xi_{\nu}-0\right)-\Phi_{j}^{\prime \prime \prime}\left(\xi_{\nu}+0\right)\right]+\int_{0}^{\infty} F^{I V} \Phi_{j} d t .
\end{gathered}
$$

Пусть теперь $f-$ произвольная функция из $\Omega_{4}[0, \infty)$. Положив $F(t)=f\left(t+t_{0}\right), t_{0}>0$, из (5.1) получаем

$$
\left|f^{(j)}\left(t_{0}\right)\right| \leq\left|\Phi_{j}^{\prime \prime \prime}(0)\right|+\left|\Phi_{j}^{\prime \prime \prime}(a-0)-\Phi_{j}^{\prime \prime \prime}(a+0)\right|+\sum_{\nu=0}^{\infty}\left|\Phi_{j}^{\prime \prime \prime}\left(\xi_{\nu}-0\right)-\Phi_{j}^{\prime \prime \prime}\left(\xi_{\nu}+0\right)\right|+48 \int_{0}^{\infty}\left|\Phi_{j}\right| d t
$$

для $j=\overline{1,3}$. Это означает, что для произвольной функции $f \in \Omega_{4}[0, \infty)$ при $j=\overline{1,3}$ верно неравенство

$$
\left\|f^{(j)}\right\| \leq\left|\Phi_{j}^{\prime \prime \prime}(0)\right|+\left|\Phi_{j}^{\prime \prime \prime}(a-0)-\Phi_{j}^{\prime \prime \prime}(a+0)\right|+\sum_{\nu=0}^{\infty}\left|\Phi_{j}^{\prime \prime \prime}\left(\xi_{\nu}-0\right)-\Phi_{j}^{\prime \prime \prime}\left(\xi_{\nu}+0\right)\right|+48 \int_{0}^{\infty}\left|\Phi_{j}\right| d t .
$$

Пусть $\varphi-$ функция из леммы 5. Положим $F(t)=\varphi(t)$. Тогда из (5.1) следует, что

$$
\begin{gathered}
(-1)^{j+1} \varphi^{(j)}(0)=-\varphi(0) \Phi_{j}^{\prime \prime \prime}(0)+\varphi(a)\left[\Phi_{j}^{\prime \prime \prime}(a-0)-\Phi_{j}^{\prime \prime \prime}(a+0)\right] \\
+\sum_{\nu=0}^{\infty} \varphi\left(\xi_{\nu}\right)\left[\Phi_{j}^{\prime \prime \prime}\left(\xi_{\nu}+0\right)-\Phi_{j}^{\prime \prime \prime}\left(\xi_{\nu}-0\right)\right]+\int_{0}^{\infty} \varphi^{I V}(t) \Phi_{j}(t) d t \\
=\left|\Phi_{j}^{\prime \prime \prime}(0)\right|+\left|\Phi_{j}^{\prime \prime \prime}(a-0)-\Phi_{j}^{\prime \prime \prime}(a+0)\right|+\sum_{\nu=0}^{\infty}\left|\Phi_{j}^{\prime \prime \prime}\left(\xi_{\nu}+0\right)-\Phi_{j}^{\prime \prime \prime}\left(\xi_{\nu}-0\right)\right|++48 \int_{0}^{\infty}\left|\Phi_{j}(t)\right| d t .
\end{gathered}
$$

Соотношение (5.2) принимает вид $\left\|f^{(j)}\right\| \leq(-1)^{j+1} \varphi^{(j)}(0)$.

Доказанную теорему можно сформулировать следующим образом. 
Теорема 1. Справедливы равенства

$$
\begin{array}{r}
\mu_{41}=\varphi^{\prime}(0)=\frac{4\left(2 \alpha_{0}^{8}-2+\left(2-\alpha_{0}^{4}+\alpha_{0}^{8}\right) \sqrt{1+\alpha_{0}^{4}}\right)}{\alpha_{0}^{9}}, \\
\mu_{42}=-\varphi^{\prime \prime}(0)=\frac{4\left(\alpha_{0}^{8}+3 \alpha_{0}^{4}+6+6\left(\alpha_{0}^{4}-1\right) \sqrt{1+\alpha_{0}^{4}}\right)}{\alpha_{0}^{6}}, \\
\mu_{43}=\varphi^{\prime \prime \prime}(0)=24 \frac{2 \sqrt{1+\alpha_{0}^{4}}+\alpha_{0}^{4}-1}{\alpha_{0}^{3}} .
\end{array}
$$

Функиия $\varphi(t)$ является экстремальной для неравенств (1.2).

Отметим теперь, что для вычисления величин $\mu_{4} j, j=\overline{1,3}$, необходимо на кривой $U M$ уметь находить точку $z_{0}$ такую, что при $\forall n \in \mathbb{N}(S K)^{n} z_{0} \in \bar{G}$ (см. рис. 3).

Покажем теперь, что экстремальная функция для неравенств (1.2) единственна с точностью до знака.

Теорема 2. Для каждого $j=\overline{1,3}$ справедливо следующее утверждение. В классе функций $\Omega_{4}[0, \infty)$ с точностью до знака существует лищь одна функиия $f$, удовлетворяющая равенству

$$
\left\|f^{(j)}\right\|=\mu_{4 j}
$$

Д о к а з а т е л ь с т в о. Покажем, что оценка (5.4) достигается только в точке $t=0$. Для этого отдельно рассмотрим три случая.

1. Оценка (5.4) достигается в некоторой точке $t_{0}>0$, т. е. $\left|f^{(j)}\left(t_{0}\right)\right|=\mu_{4 j}$.

2. Существует последовательность $\left\{t_{n}\right\}_{n=1}^{\infty}$ точек $t_{n} \rightarrow \infty$ при $n \rightarrow \infty$ такая, что $\lim _{n \rightarrow \infty}\left|f^{(j)}\left(t_{n}\right)\right|=\mu_{4 j}$.

3. $\left|f^{(j)}(0)\right|=\mu_{4 j}$.

1. Рассмотрим первый случай. Пусть функция $f(t)$ такова, что для нее имеет место равенство

$$
(-1)^{j+1} f^{(j)}\left(t_{0}\right)=\mu_{4 j}
$$

(если надо, у функции $f(t)$ изменим знак). $\mathrm{K}$ функции $F(t)=f\left(t+t_{0}\right)$ применим равенство (5.1). Имеем

$$
\begin{aligned}
& (-1)^{j+1} f^{(j)}\left(t_{0}\right)=\left|\Phi_{j}^{\prime \prime \prime}(0)\right|+\left|\Phi_{j}^{\prime \prime \prime}(a-0)-\Phi_{j}^{\prime \prime \prime}(a+0)\right|+\sum_{\nu=0}^{\infty}\left|\Phi_{j}^{\prime \prime \prime}\left(\xi_{\nu}-0\right)-\Phi_{j}^{\prime \prime \prime}\left(\xi_{\nu}+0\right)\right| \\
& +48 \int_{0}^{\infty}\left|\Phi_{j}(t)\right| d t=-f\left(t_{0}\right) \Phi_{j}^{\prime \prime \prime}(0)+f\left(t_{0}+a\right)\left[\Phi_{j}^{\prime \prime \prime}(a-0)-\Phi_{j}^{\prime \prime \prime}(a+0)\right] \\
& +\sum_{\nu=0}^{\infty} f\left(t_{0}+\xi_{\nu}\right)\left[\Phi_{j}^{\prime \prime \prime}\left(\xi_{\nu}-0\right)-\Phi_{j}^{\prime \prime \prime}\left(\xi_{\nu}+0\right)\right]+\int_{0}^{\infty} f^{I V}\left(t_{0}+t\right) \Phi_{j}(t) d t .
\end{aligned}
$$

Сравнивая соответствующие слагаемые, получаем

$$
\begin{aligned}
f\left(t_{0}\right) & =-1, \quad f\left(t_{0}+a\right)=+1, \quad f\left(t_{0}+\xi_{\nu}\right)=(-1)^{\nu+1}, \quad \nu=\overline{0, \infty}, \\
f^{I V}\left(t_{0}+t\right) & =-48, \quad \text { если } t \in\left[0, x_{1}\right], \\
f^{I V}\left(t_{0}+t\right) & =(-1)^{\nu+1} \cdot 48, \quad \text { если } t \in\left(x_{\nu}, x_{\nu+1}\right), \quad \nu=\overline{1, \infty} .
\end{aligned}
$$


Для $t \in\left[0, x_{1}\right]$ имеем равенство $f\left(t+t_{0}\right)=-2(t-b)^{4}+4 A_{0}(t-b)^{3}+2 B_{0}(t-b)^{2}-1$. Поскольку $f\left(t_{0}\right)=-1$ и $f\left(t_{0}+a\right)=1$, получаем, что

$$
\begin{aligned}
F(t)=f\left(t+t_{0}\right) & =-2(t-b)^{4}+4 \frac{1-\alpha_{0}^{4}}{\alpha_{0}^{3}}(t-b)^{3}+2 \frac{3-\alpha_{0}^{4}}{\alpha_{0}^{2}}(t-b)^{2}-1 \\
& =-1+\mu_{41} t-\frac{\mu_{42}}{2 !} t^{2}+\frac{\mu_{43}}{3 !} t^{3}-2 t^{4}
\end{aligned}
$$

(в силу (5.5)). Это означает, что в окрестности точки $t_{0}$ справедливо равенство

$$
f\left(t_{0}+t\right)=-1+\mu_{41} t+\overline{\bar{o}}\left(t^{2}\right)
$$

где $\mu_{41} \neq 0$. Но тогда для некоторых $t<t_{0}$ справедливо неравенство $f(t)<-1$, что невозможно, так как $\|f\|=1$.

2. Изменив, если надо, знак функции $f(t)$ или выбрав подпоследовательность из $\left\{t_{n}\right\}_{n=1}^{\infty}$, будем считать выполненными соотношения

$$
\lim _{n \rightarrow \infty} f^{(j)}\left(t_{n}\right)=\varphi^{(j)}(0), \quad j=\overline{1,3}
$$

Взяв в качестве $F$ функцию $F(t)=f\left(t_{n}+t\right)$ и воспользовавшись равенством $(5.1)$, получим

$$
\begin{aligned}
f\left(t_{n}\right) & =-1+\delta_{n}^{\prime}, \\
f\left(t_{n}+a\right) & =1+\delta_{n}^{\prime \prime} \\
f\left(t_{n}+b\right) & =-1+\delta_{n}^{\prime \prime \prime}, \quad \delta_{n}^{\prime}, \delta_{n}^{\prime \prime}, \delta_{n}^{\prime \prime \prime} \rightarrow 0 \text { при } n \rightarrow \infty .
\end{aligned}
$$

Проводя рассуждения, аналогичные предыдущим, можно доказать, что

$$
f\left(t_{n}+t\right)=-1+\mu_{41} t+\overline{\bar{o}}\left(t^{2}\right)+\tilde{\delta_{n}},
$$

где $\overline{\bar{o}}\left(t^{2}\right)$ равномерно относительно $t \in[0, b]$, а $\tilde{\delta_{n}} \rightarrow 0$ при $n \rightarrow \infty$. Отсюда при достаточно больших $n$ и достаточно малых отрицательных $t$ будем иметь $f\left(t_{n}+t\right)<-1$, что невозможно.

3. Рассмотрим, наконец, последний случай. Изменив в случае необходимости знак функции $f(t)$, по аналогии с первым случаем имеем

$$
f(0)=-1, \quad f(a)=1, \quad f(b)=-1, \quad f^{I V}(t)=\varphi^{I V}(t)
$$

Для $t \in[0, b]$

$$
f(t)=-1+\frac{f^{\prime \prime}(b)}{2 !}(t-b)^{2}+\frac{f^{\prime \prime \prime}(b)}{3 !}(t-b)^{3}-2(t-b)^{4} .
$$

С учетом условий $f(0)=-1$ и $f(a)=1$ отсюда получаем, что для $t \in[0, b] f(t)=\varphi(t)$. Из этого равенства и соотношения (5.6) вытекает, что $f(t)=\varphi(t)$ при всех $t \in[0, \infty)$.

Следствие 2. На дуге $U M$ (см. лемму 3) существует только одна точка $z_{0}$, обладающая свойством $(S K)^{n} z_{0} \in \bar{G}$ при всех натуральных $n$.

Д о к а з а т е л ь с т в о. Если бы существовала другая точка с теми же свойствами, то можно было бы построить (см. леммы 5 и 8 ) еще одну функцию $\tilde{\varphi}$ и еще один набор функций $\tilde{\Phi}_{j}, j=\overline{1,3}$. По теореме 1 функция $\tilde{\varphi} \in \Omega[0 ; \infty)$ и будет экстремальной в задачах $(1.2)$. 


\section{6. Алгоритм вычисления значения $\alpha_{0}$}

Из следствия к теореме 2 вытекает, что дополнение $Y_{1} \cup Y_{2}$ до открытой дуги $U M$ состоит из одной точки $z_{0}$. Это значит, что $Y_{1}$ и $Y_{2}$ являются интервалами на дуге $U M$. Это дает нам способ вычисления параметра $\alpha_{0}$, а именно выберем какую-нибудъ точку $z_{1}$ на дуге $M U u$ вычислим $\gamma_{n}^{(1)}=(S K)^{n} z_{1}$. Если при некотором $n$ точка $\gamma_{n}^{(1)}$ выходит за пределы $\bar{G}$ и при этом оказывается в правой (соответственно в левой) полуплоскости, то в качестве точки $z_{2}$ выбираем середину дуги $z_{1} U$ (соответственно $\left.z_{1} M\right)$. Таким образом, строим последовательность точек $\left\{z_{n}\right\}$, сходящуюся $\kappa z_{0}$.

Алгоритм дает следующие результаты:

$$
\alpha_{0} \approx 1.00674052
$$

$C_{4,1}\left(\mathbb{R}_{+}\right)=\frac{\mu_{41}}{\sqrt[4]{48}} \approx 4.28850981, C_{4,2}\left(\mathbb{R}_{+}\right)=\frac{\mu_{42}}{\sqrt{48}} \approx 5.75037058, C_{4,3}\left(\mathbb{R}_{+}\right)=\frac{\mu_{43}}{(\sqrt[4]{48})^{3}} \approx 3.70803527$.

Автор выражает глубочайшую благодарность рецензентам за конструктивные замечания, повлиявшие на более четкое и ясное изложение статьи.

\section{СПИСОК ЛИТЕРАТУРЫ}

1. Landau E. Einige Ungleichungen für zweimal differentierbare Funktionen // Proc. London Math. Soc. (2). 1913. Vol. 13. P. 43-49. doi: 10.1112/plms/s2-13.1.43.

2. Колмогоров А.Н. О неравенствах между верхними гранями последовательных производных произвольной функции на бесконечном интервале // Избр. тр. Математика, механика. М.: Наука, 1985. С. 252-263. (Уч. зап. Моск. ун-та. Математика, кн. 3. 1939. Т. 30. С. 3-16.)

3. Маторин А.П. О неравенствах между наибольшими значениями абсолютных величин функции и ее производных на полупрямой // Укр. мат. журн. 1955. Т. 7. С. 262-266.

4. Стечкин С.Б. О неравенствах между верхними гранями производных произвольной функции на полуоси // Мат. заметки. 1967. Т. 1, вып. 6. С. 665-674.

5. Schoenberg I.J., Cavaretta A. Solution of Landau's problem, concerning higher derivatives on halfline // M.R.C. Technical Summary Report. 1970. No. 1050. Madison Wis., 1970.

6. Schoenberg I.J., Cavaretta A. Solution of Landau's problem, concerning higher derivatives on halfline // Proc. Conf. Approx. Theory (Varna 1970). Sofia, 1972. P. 297-308.

7. Стечкин С.Б. Наилучшее приближение линейных операторов // Мат. заметки. 1967. Т. 1, вып. 2. C. $137-148$.

8. Арестов В.В. Приближение неограниченных операторов ограниченными и родственные экстремальные задачи // Успехи мат. наук. 1996. Т. 51, вып. 6. С. 89-124. doi: https://doi.org/10.4213/rm1019.

9. Тимофеев В.Г. Колмогоровские оценки в равномерной метрике на полуоси через функцию и ее пятую производную // Математика, механика: сб. тр. Саратов: Изд. Сарат. гос. ун-та, 2000. № 2. C. $122-125$.

10. Тимофеев В.Г. Об одном специальном отображении // Изв. Сарат. ун-та. Новая серия. Сер. Математика. Механика. Информатика. 2011. Т. 11, вып. 3. С. 54-60.

11. Кудрявцев Л.Д. Дифференцируемые отображения $n$-мерных областей и гармонические отображения плоских областей // Заседание Моск. мат. об-ва. Успехи мат. наук. 1954. Т. 9, вып. 2 (60). С. 207-209.

12. Кудрявцев Л.Д. О вариации отображений областей // Математические вопросы теории функций и отображений: сб. докл. коллоквиума по теории квазиконформных отображений и ее обобщениям. Вып. І. (Донецк, сентябрь 1968 г.) Киев: Изд-во "Наукова думка", 1969. С. 34-108.

Тимофеев Владимир Григорьевич

канд. физ.-мат. наук, доцент

Саратовский национальный исследовательский государственный университет им. Н.Г. Чернышевского,

г. Саратов

e-mail: timofeevvg48@gmail.com
Поступила 9.12.2018

После доработки 6.05.2019

Принята к публикации 20.05.2019 


\section{REFERENCES}

1. Landau E. Einige Ungleichungen für zweimal differentierbare Funktionen. Proc. London Math. Soc. (2), 1913, vol. 13, pp. 43-49. doi: 10.1112/plms/s2-13.1.43.

2. Kolmogorov A.N. On inequalities between upper bounds of consecutive derivatives of an arbitrary function defined on an infinite interval. Selected works. Mathematics and Mechanics. Moscow: Nauka Publ., 1985, pp. 252-263. (Moskov. Gos. Univ., Uchenye Zap. (Mat. 3), 1939, vol. 30, pp. 3-16) (in Russian).

3. Matorin A.P. On inequalities between the maxima of the absolute values of a function and its derivatives on a half-line. Amer. Math. Soc. Transl. Ser. 2, 1958, vol. 8, pp. 13-17.

4. Stechkin S.B. Inequalities between upper bounds of the derivatives of an arbitrary function on the half-line. Math. Notes, 1967, vol. 1, no. 6, pp. 442-447. doi: 10.1007/BF01093072 .

5. Schoenberg I.J., Cavaretta A. Solution of Landau's problem, concerning higher derivatives on half-line. M.R.C. Technical Summary Report. No. 1050. Madison Wis., 1970.

6. Schoenberg I.J., Cavaretta A. Solution of Landau's Problem, concerning higher derivatives on half-line. In: Proc. Conf. on Approximation Theory (Varna 1970). Sofia, 1972, pp. 297-308.

7. Stechkin S.B. Best approximation of linear operators. Math. Notes, 1967, vol. 1, no. 2, pp. 91-99. doi: $10.1007 /$ BF01268056 .

8. Arestov V.V. Approximation of unbounded operators by bounded operators and related extremal problems. Russ. Math. Surv., 1996, vol. 51, no. 6, pp. 1093-1126. doi: 10.1070/RM1996v051n06ABEH003001.

9. Timofeev V.G. Kolmogorov-type estimates in a uniform metric on the half-line by the function and its fifth derivative. In: Mathematics. Mechanics: Collection of Scientific Papers, Saratov: Saratov Univ. Publ., 2000, no. 2, pp. 122-125.

10. Timofeev V.G. On one special mapping. Izv. Saratov Univ. (N.S.), Ser. Math. Mech. Inform., 2011, vol. 11, no. 3 (1), pp. 54-60 (in Russian).

11. Kudryavtsev L.D. Differentiable mappings of $n$-dimensional domains and harmonic mappings of flat domains. In: Meetings of the Moscow Mathematical Society. Uspekhi Mat. Nauk, 1954, vol. 9, no. 2 (60), pp. 207-213 (in Russian).

12. Kudryavtsev L.D. The variation of mappings of regions. In: Metrical Questions of the Theory of Functions and Mappings, no. 1 (Proc. Colloq. on the Theory of Quasiconformal Mappings and its Generalizations, Donetsk, 1968). Kiev: Naukova Dumka Publ., 1969, pp. 34-108 (in Russian).

Received December 9, 2018

Revised May 6, 2019

Accepted May 20, 2019

Vladimir Grigor'evich Timofeev, Cand. Sci. (Phys.-Math.), Saratov State University, Saratov, 410012 Russia, e-mail: timofeevvg48@gmail.com .

Cite this article as: V.G. Timofeev. N.P. Kuptsov's method for the construction of an extremal function in an inequality between uniform norms of derivatives of functions on the half-line, Trudy Instituta Matematiki i Mekhaniki URO RAN, 2019, vol. 25, no. 2, pp. 220-239. 